\title{
Deconvolution of Continuous Borehole Temperature Logs: Example from the Greenland GISP2 Icecore Hole
}

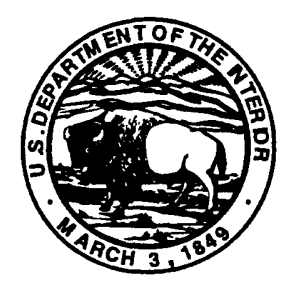

Open-File Report 94-254

This report is preliminary and has not been reviewed for conformity with U.S. Geological Survey editorial standards. Use of brand names is for descriptive purposes and does not constitute endorsement by the U.S. Geological Survey.

${ }^{1}$ U.S. Geological Survey, Denver, Colorado

${ }^{2}$ U.S. Geological Survey, Menlo Park, California 


\begin{abstract}
Measurements of borehole temperatures obtained from a continuously moving thermistor probe are a convolution of the actual borehole temperatures with the finite thermal response function of the temperature measurement system. To estimate actual temperatures from the measurements requires deconvolution, an operation that can magnify noise. We discuss the use of optimal (Wiener) filtering for noise reduction, followed by an exact deconvolution using serial division. We test the method using synthetic data and then apply it to measurements made in May 1992 at the GISP2 icecore hole in Greenland. After optimal filtering of the 1992 GISP2 measurements, the data power spectrum falls to $10 \%$ of maximum as frequencies drop below $0.004 \mathrm{~Hz}$ (periods of 250 seconds, equivalent to about $20 \mathrm{~m}$ in depth at a logging velocity of about $7.4 \mathrm{~cm} / \mathrm{s}$ ). The noise at higher frequencies (shorter wavelengths) is probably a result of noise caused by the mechanical slip-ring that provides electrical connection to the thermister cable.
\end{abstract}




\section{Contents}

1 INTRODUCTION 4

2 TEMPERATURE MEASUREMENT 4

2.1 Response time of thermistor probe .............. 5

3 TEMPERATURE DECONVOLUTION 6

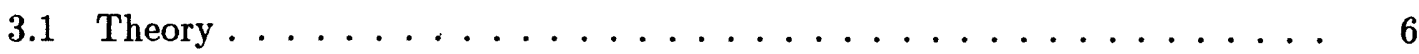

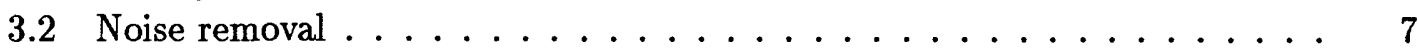

3.3 Discrete deconvolution by division .............. 8

3.4 Implications of discrete interval for deconvolution . . . . . . . . . 8

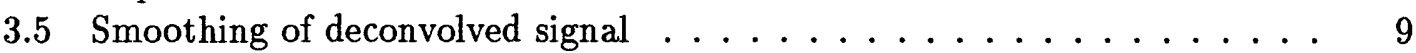

3.6 Expected downhole variation of signal frequency content . . . . . . . . 9

4 EXAMPLES 9

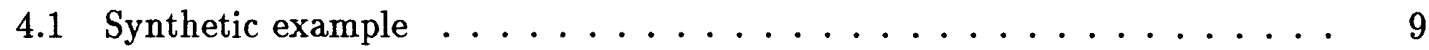

4.2 Deconvolution of measured temperatures in GISP2 borehole . . . . . . . 15

5 CONCLUSION 20

6 ACKNOWLEDGEMENTS 25

7 APPENDIX A: MEASUREMENT OF PROBE RESPONSE FUNC$\begin{array}{ll}\text { TION } & 28\end{array}$

8 APPENDIX B: COMPUTER CODES 35

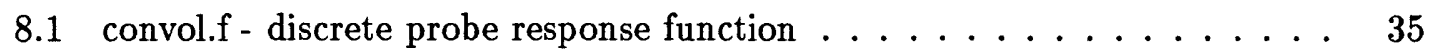

8.2 response.f - discrete probe response function $\ldots \ldots \ldots 36$

8.3 Example IDL program for optimal filtering $\ldots \ldots \ldots \ldots \ldots$

8.4 simdecon.f - exact discrete deconvolution . . . . . . . . . . . . 39

\section{List of Tables}

1 Temperature probe time constants ..................... 6

2 Optimal filter functions - signal plus noise .............. 12 


\section{List of Figures}

1 Synthetic data and convolution. . . . . . . . . . . . 10

2 Detail plot of synthetic data and noisy convolution. . . . . . . . . 11

3 Power spectrum and optimal filter definition: before deconvolution . . . . 12

4 Power spectrum and optimal filter definition: after deconvolution . . . . 13

5 Detailed comparison of synthetic data, convolution, and deconvolution . . 14

6 Error histograms - synthetic test case. . . . . . . . . . . . 15

7 May 1992 GISP2 measured temperatures. . . . . . . . . . . . 16

8 Data power spectrum and optimal filter definition. . . . . . . . . 17

9 Optimal smoothing filter - May 1992 GISP2 data smoothing. . . . . . . 18

10 Histogram of data noise (data - smoothed data). . . . . . . . . . 19

11 Deconvolved power spectrum and second optimal filter definition. . . . . 20

12 Optimal smoothing filter - May 1992 GISP2 deconvolution smoothing. . . 21

13 Detailed comparison of raw, smoothed, and deconvolved data. . . . . . 22

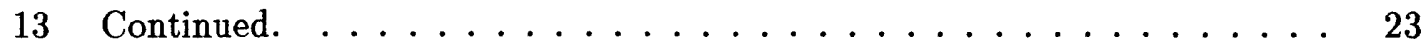

14 May 1992 GISP2 difference of smoothed data and deconvolution. . . . . . 24

A1 Thermister probe response in stirred alcohol. . . . . . . . . . . 29

A2 Thermister probe response in unstirred alcohol. . . . . . . . . . 30

A3 Thermister probe response in a still ice bath. . . . . . . . . 31

A4 Thermister probe response in still Jet A fuel. . . . . . . . . . . . 32

A5 Depth verses temperature, fluid entry at GISP2, May $1992 \ldots \ldots \ldots \ldots 33$

A6 Thermister probe response at GISP2, May 1992. . . . . . . . . . 34 


\section{INTRODUCTION}

Temperatures measured in boreholes contain a record of the temperature change history at the Earth's surface (Thomson, 1862; Birch, 1948; Lachenbruch and Marshall, 1986). Because of the smoothing effects of heat conduction and convection processes, this climate record loses fidelity with increasing depth. One way to compensate for this inevitable loss of resolution with depth, is to collect the most accurate borehole temperature record possible. Backus-Gilbert analysis of the borehole temperature inverse problem (Clow, 1992), indicates that recovery of climate history is optimized by increasing measurement resolution to $1 \mathrm{mK}\left(0.001^{\circ} \mathrm{C}\right)$. Also, $1 \mathrm{mK}$ accuracy allows for better detection of climaterelated transient temperature changes in repeat borehole logging (Lachenbruch, 1994). To achieve this measurement accuracy, an experimental borehole logging system has been developed (Clow and others, 1995) and used to collect data in the GISP2 climate research hole drilled into the Greenland icecap (Boulton, 1993; Morrison, 1993). The data were collected in a continuous logging mode as the thermistor probe descended at about 7.6 $\mathrm{cm} / \mathrm{s}$ into the icecore hole. The corehole is filled with liquid n-butyl acetate to prevent collapse of the hole. This paper documents our processing of these data to remove the random $\pm 1.5 \mathrm{mK}$ mechanically produced "slip-ring" noise from the measurements and our scheme for deconvolution to remove the effects of the finite (approx. 15-sec time constant) time response of the temperature measurement system.

\section{TEMPERATURE MEASUREMENT}

The measurement of temperatures in boreholes has evolved in several phases (e.g., Jessop, 1990, p. 23). The earliest measurements were made by lowering glass thermometers with long time constants to depth, allowing them to equilibrate, and then quickly withdrawing them and recording the temperature reading. Modern borehole temperature measurement generally involves monitoring the resistance of an electrical thermistor probe that is lowered into a borehole (Beck and Balling, 1988). Two modes of operation are in use: start/stop and continuous logging. In the start/stop mode the probe is stationary for each temperature measurement; the probe is allowed to reach sufficient thermal equilibrium to meet the measurement accuracy required by the study. Reported times for a single start/stop measurement range from more than 10 minutes (Costain, 1970) to times as short as $30 \mathrm{~s}$ (Chisholm and Chapman, 1992). In the continuous mode (Costain, 1970; Conaway, 1977; Conaway and Beck, 1977), the resistance of the thermistor probe is recorded at discrete intervals (either of time or distance, depending on the system used) as the probe is lowered continuously into the borehole.

If the response time of the probe is small relative to the discrete measurement interval then temperatures measured in the continuous mode will be a good representation of the temperature at each measurement point. If, however, the probe time constant is on the order of the measurement interval or more, then the measured temperatures will be a smoothed version of the actual temperature distribution. For some studies, such as the determination of average thermal gradients for calculation of heat flow, this smoothing does not present a problem. For other work, such as detailed formation conductivity estimation or inversion for near-surface temperature variation, the smoothed record is a 
disadvantage.

\subsection{Response time of thermistor probe}

The response of a thermistor probe system to a step change in temperature, $\Delta T$, has been modeled by (Costain, 1970; Nielsen and Balling, 1984)

$$
s(t)=T_{\infty}+\Delta T \exp \left(-\frac{t-t_{o}}{\tau}\right)
$$

where $T_{\infty}$ is the new equilibrium temperature, $\tau$ is the time constant of the system, $t_{o}$ is the time delay before the system begins to sense the temperature change, and $t$ is time. If equation 1 is rewritten as

$$
t=t_{o}-\tau \ln \left(\frac{s(t)-T_{\infty}}{\Delta T}\right)
$$

then $\tau$ and $t_{o}$ can be determined as the slope $(\tau)$ and intercept $\left(t_{o}\right)$ of a linear regression of time verses the function $\ln \left[\left(s(t)-T_{\infty}\right) / \Delta T\right]$.

The time constant and time delay will depend on the thermal properties and design of the probe system as well as the thermal properties and temperature of the fluid in contact with the probe. Time constants reported in the literature range from 7 seconds (Chisholm and Chapman, 1992) to 15 seconds (Costain, 1970; Conaway, 1977) for thermistor bead probes and up to 18.7 seconds (Nielson and Balling, 1984) for a quartz oscillator thermometer. Chisholm and Chapman (1992) report a $7 \mathrm{~s}$ time constant for a logging system based on the same probe (Fenwal 212E) as used in our system. A recent study (Sawyer and others, 1994) of data collected on the Ocean Drilling Program suggests a probe time constant of more than $500 \mathrm{~s}$ (however, no physical explanation was found for this empirical result). Reported time delays range from negligible (Sass and others, 1971) to 0.2 seconds (Conaway, 1977) for thermistor bead probe systems and up to 3.8 seconds (Nielson and Balling, 1984) for a quartz oscillator thermometer system. Sawyer and others (1994) interpreted a time delay of $125 \mathrm{~s}$ for their probe system. Our measurements (appendix A) show a range of time constants for our Fenwal $212 \mathrm{E}$ thermistor bead probe system (Table 1). We have not experienced any noticeable time delay with our logging system. Measuring the probe system response in the same physical conditions as encountered in the borehole is important.

Because the measurement system has a finite time constant, any measured temperature is an averaged value based on the temperatures encountered by the probe during the last several time constants; temperatures measured by a probe in continuous motion down a borehole are a smoothed version of the actual temperature variation.

In addition to the physical response of the temperature probe system, the accuracy of time and depth information for each measurement are important to reconstruction of the actual temperatures. As discussed in the next section, temperature measurements at regular time intervals are required for deconvolution of the probe response. If the logging system does not take measurements at regular time intervals then some form of interpolation will be required to estimate temperatures at regular time intervals. This interpolation would contribute to uncertainty in the recovered values. 
Table 1: Temperature probe time constants

\begin{tabular}{llllll}
\hline probe & fluid & $t\left({ }^{\circ} \mathrm{C}\right)$ & $\tau(\mathrm{s})$ & comments & ref \\
\hline Fenwal 212E & alcohol & 13 & 2.7 & stirred & Appendix A \\
Fenwal 212E & alcohol & 13 & 3.8 & still & Appendix A \\
Fenwal 212E & water & $?$ & 7 & still & Chisholm and Chapman, 1992 \\
Fenwal 212E & water & 0 & 7.2 & still, ice bath & Appendix A \\
Fenwal 212E & DFA & $?$ & 10 & Alaska field test & Clow, pers. comm. \\
Fenwal 212E & Jet-A & 13 & 14.7 & still & Appendix A \\
Fenwal 212E & n-butyl & -31 & 15.0 & GISP2 field test & Appendix A \\
& acetate & & & & \\
thermistor & water & $?$ & 15 & & Costain, 1970 \\
qtz oscillator & water & $?$ & 18.7 & & Nielson and Balling, 1984 \\
ODP probe & water & $?$ & 500 & no physical basis & Sawyer and others, 1994 \\
\hline
\end{tabular}

\section{TEMPERATURE DECONVOLUTION}

Several workers have discussed the problem of deconvolution of measurement system response from borehole temperatures measured with a moving probe (Costain, 1970; Conaway, 1977; Nielsen and Balling, 1984; Sawyer and others, 1994). The initial studies (Costain, 1970; Conaway, 1977) focussed on developing a combined gradient, deconvolution, and smoothing operator for determination of temperature gradients from continuously measured profiles. The combined operator approach is efficient for quick processing, but doesn't allow separate noise analysis. Nielsen and Balling (1984) use a Backus-Gilbert approach to study the trade-off between resolution and accuracy in deconvolution. Their method involves selection of an a priori averaging function and determination of a set of smoothing coefficients (one for each data point). In recent communication, Nielsen (written communication, 1993) suggests breaking the problem into separate smoothing and deconvolution steps; that is the approach we take here.

\subsection{Theory}

The finite time response of the thermistor probe system has the effect of smoothing measured temperatures. The word "convolution" describes this smoothing; it is defined as (Bracewell, 1986, p. 24) "the action of an observing instrument when it takes a weighted mean of some physical quantity over a narrow range of some variable." The following mathematical formulation for the measurement process contains the assumption that we know the mathematical form of the impulse response of our temperature probe system and that it is independent of temperature (i.e., that we have a linear system). The form of the probe impulse response to a step change in temperature (equation 1) and the related form for an impulse response (equation 3) have been assumed by previous workers (i.e., Costain, 1970; Conaway, 1977; Nielsen and Balling, 1984). Our limited experience with measurement of the probe time constant (see Appendix A) suggests that this form is good 
for the first part of the temperature response, but breaks down at longer times after the temperature step or impulse. We plan to investigate this further.

If we assume the smoothing response for a temperature impulse is given by (Nielson and Balling, 1984)

$$
\begin{gathered}
h(t)=(1 / \tau) \exp \left(-\frac{t-t_{o}}{\tau}\right), t \geq t_{o} \\
h(t)=0, t<t_{o}
\end{gathered}
$$

then, for a general temperature function, $d(t)$, the smoothing can be expressed as the convolution (Nielson and Balling, 1984):

$$
m(t)=\int_{-\infty}^{\infty} d(\mu) h(t-\mu) d \mu
$$

The problem is to deconvolve the measured temperature function, $m(t)$, to obtain the data, $d(t)$.

The convolution of the probe system response with actual temperature is a continuous process, but our measurements are made at discrete intervals in the presence of noise. If we had noise-free, continuous measurements we could use Fourier transform theory for deconvolution; it becomes a division in the Fourier domain (Bracewell, 1986). Because of the discrete time spacing of our measurements, however, we have an incomplete record. Any attempt to recover the actual temperature distribution is limited to the frequencies available in our discrete measurements. The presence of noise in our measurements causes our incomplete record to also be inaccurate. The process of deconvolution, an un-smoothing, will amplify noise. The treatment of this noise is a key problem in deconvolution of our measurements.

\subsection{Noise removal}

Since deconvolution amplifies noise, we must try to remove noise from our measured signal before attempting to deconvolve. Noise in borehole temperature measurements may result from electical and mechanical effects such as ground current loops, thermal electromagnetic forces, triboelectic effects, space charge effects, interconductor leakage, digital resolution, irregular electrical contact in the slip-ring assembly, or other mechanical inaccuracies. Noise may also result from random physical processes such as convection within the fluid of the borehole. The noise spectrum of any given temperature log may then be a function of both the instrumental setup and the physical conditions in the borehole. Thus, noise should be analyzed separately for each instrument and borehole.

In the absence of independent knowledge of the noise sources, it is often possible to construct a noise model by examination of the power spectrum of the measured data (Press and others, 1992; section 13.3). An optimal (Wiener) filter for noise removal in the frequency domain can be defined as (Press and others, 1992):

$$
\Phi(f)=\frac{|S(f)|^{2}}{|S(f)|^{2}+|N(f)|^{2}}
$$

where $S$ and $N$ are the Fourier transforms of the modeled signal and noise components of the data respectively; $f$ is frequency. This function is equal to unity when the signal 
dominates the noise and will go to zero as the power of the noise increases. In practice (Press and others, 1992), the function may be approximated as the difference between smooth curves drawn through the calculated power spectrum of the measurements and an assumed model for the noise spectrum. Once this optimal filter is defined, noise can be removed from the measured signal by multiplication of the Fourier transform of the measured data with the optimal filter; this product is then inverse transformed to produce an approximation of the noise-free signal. The optimal filter defined here is a low-pass filter for which the frequency cutoff depends upon $\Phi$. In a later section we discuss two examples of this procedure, one applied to synthetic data, the second applied to temperatures measured in the GISP2 icecore hole in Greenland.

\subsection{Discrete deconvolution by division}

Convolution of two finite, discretely sampled functions can be represented as a serial product (Bracewell, 1986, p. 30). If discrete samples of the data function, $d_{i}$, and the response function, $h_{i}$, are given, then the discrete approximation of the convolution to form the measured function, $m_{i}$, is:

$$
m_{i}=\sum_{j=1}^{n} h_{j} d_{(i-j+1)}
$$

where $n$ is the number of terms in the response function, $h$. Note that this formulation requires knowledge of the data function, $d$, for times before the start of the experiment. One solution to this problem is to start with the system a steady-state such that $d_{i}=d_{1}$ for $i \leq 1$. To invert this discrete convolution we may employ serial division (Bracewell, 1986 , p. 35). To reconstruct the data function given the measurements and a discrete response function, we can use the following summation:

$$
d_{i}=\frac{m_{i}-\sum_{j=1}^{n-1} h_{(j+1)} d_{(i-j)}}{h_{1}}, i \geq 2
$$

Note that each recovered data value, $d_{i}$, depends on the previous $n-1$ recovered values. To recover the first $n-1$ data values, we must make an assumption about the initial state of our measurement system; we must assume values of the measurement function before the start of our experiment. To address this problem, we have chosen to allow the thermister probe to reach thermal equilibrium before moving the probe down the borehole. In that case, $m_{i}=m_{1}$ for $i \leq 0$ and $d_{1}=m_{1}$. A computer subroutine (in FORTRAN 77) that implements this method is given in Appendix B. This technique is advantageous over the standard FFT deconvolution technique because of its simplicity (it can be easily coded into a calculator or field computer) and because the beginning equilibrium condition is handled exactly.

\subsection{Implications of discrete interval for deconvolution}

Our purpose in attempting deconvolution is to undo the continuous convolution of temperature with probe response that is performed by the physical thermistor system. Even assuming that all noise could be removed from the measured signal, the use of a discrete 
deconvolution will always yield an imperfect recovery of the original signal. Depending on the frequency content of the signal, it may be necessary to interpolate values between the smoothed measurements so that we may use a better representation of the response function. For the synthetic data example discussed below, we determined empirically that an interpolation of 9 points between each measured point allows recovery, even in the presence of noise, of the original signal within a standard error acceptable for our system. For the GISP2 data, noise dominates to lower frequencies in the power spectrum of the data and no interpolation was necessary. Additional work should be done to determine a general relation between the frequency contents of the signal and noise, increased discrete deconvolution resolution, and errors introduced by excessive interpolation.

\subsection{Smoothing of deconvolved signal}

Since it is impossible to remove all noise from the measured signal before deconvolution, some noise amplification will result from deconvolution. A second application of optimal filtering can be used to clean up the deconvolved signal. In both of the examples discussed below, the noise present in the deconvolved signal is of much lower magnitude than the noise removed before deconvolution.

\subsection{Expected downhole variation of signal frequency content}

As mentioned in the introduction, the surface temperature record stored in the conductive borehole temperature profile is increasingly damped with depth. Thus, in the absense of other variations, we expect the shorter wavelengths in our temperature log to be increasingly damped with depth. In the analysis presented in this report we have analyzed the frequency content of the entire data $\log$ in one section, averaging any variation in frequency content with depth. In the case of the 1992 GISP2 data, this approach is probably justified because we don't expect any variation in the inferred noise source with depth.

\section{EXAMPLES}

\subsection{Synthetic example}

To test the accuracy of the noise reduction and deconvolution procedures we constructed a synthetic borehole temperature data set, convolved it with a reasonable probe response function and added random noise. We then performed optimal noise filtering and deconvolution and compared the recovered signal to the original synthetic signal. The results indicate that signal can be recovered to within $50 \%$ of the standard deviation of the added noise. In this example, the added noise had a standard deviation of $1 \mathrm{mK}$ and the standard deviation between the synthetic signal and the deconvolved signal is less than $0.5 \mathrm{mK}$.

The synthetic borehole temperatures were constructed by forward calculation from an assumed air temperature history. The synthetic data were constructed with a sample interval of $3.7 \mathrm{~mm}$ in depth. The long-term surface temperature intercept was set at $-12^{\circ} \mathrm{C}$ and the background geothermal gradient was set to $20^{\circ} \mathrm{C} / \mathrm{km}$. The resulting function has a smooth temperature variation with depth (Figure 1). 


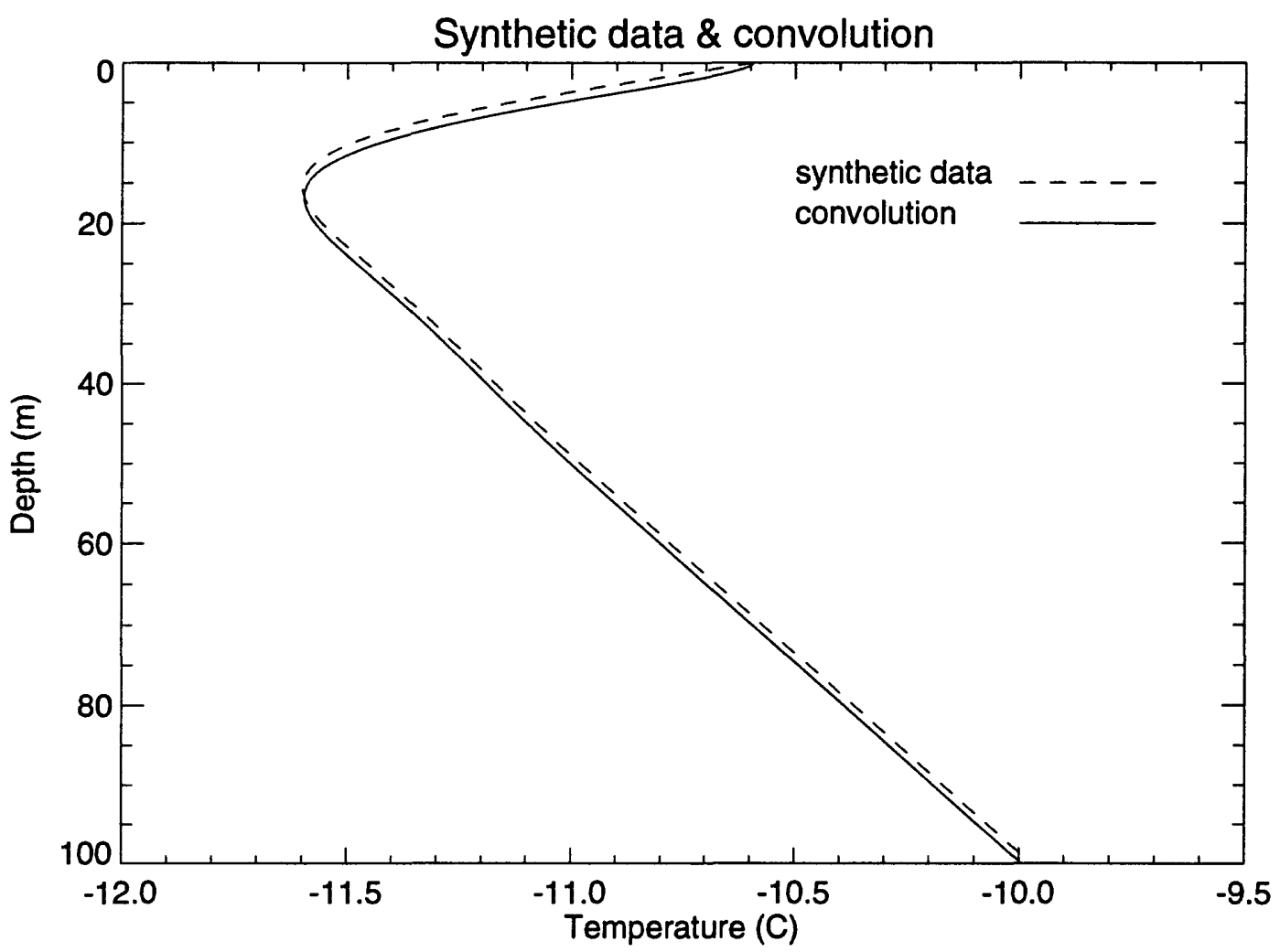

Figure 1: Synthetic data and numeric convolution with $15 \mathrm{~s}$ response function. Convolution with the finite thermistor probe response function produces a curve that is shifted down the borehole and smoothed. 


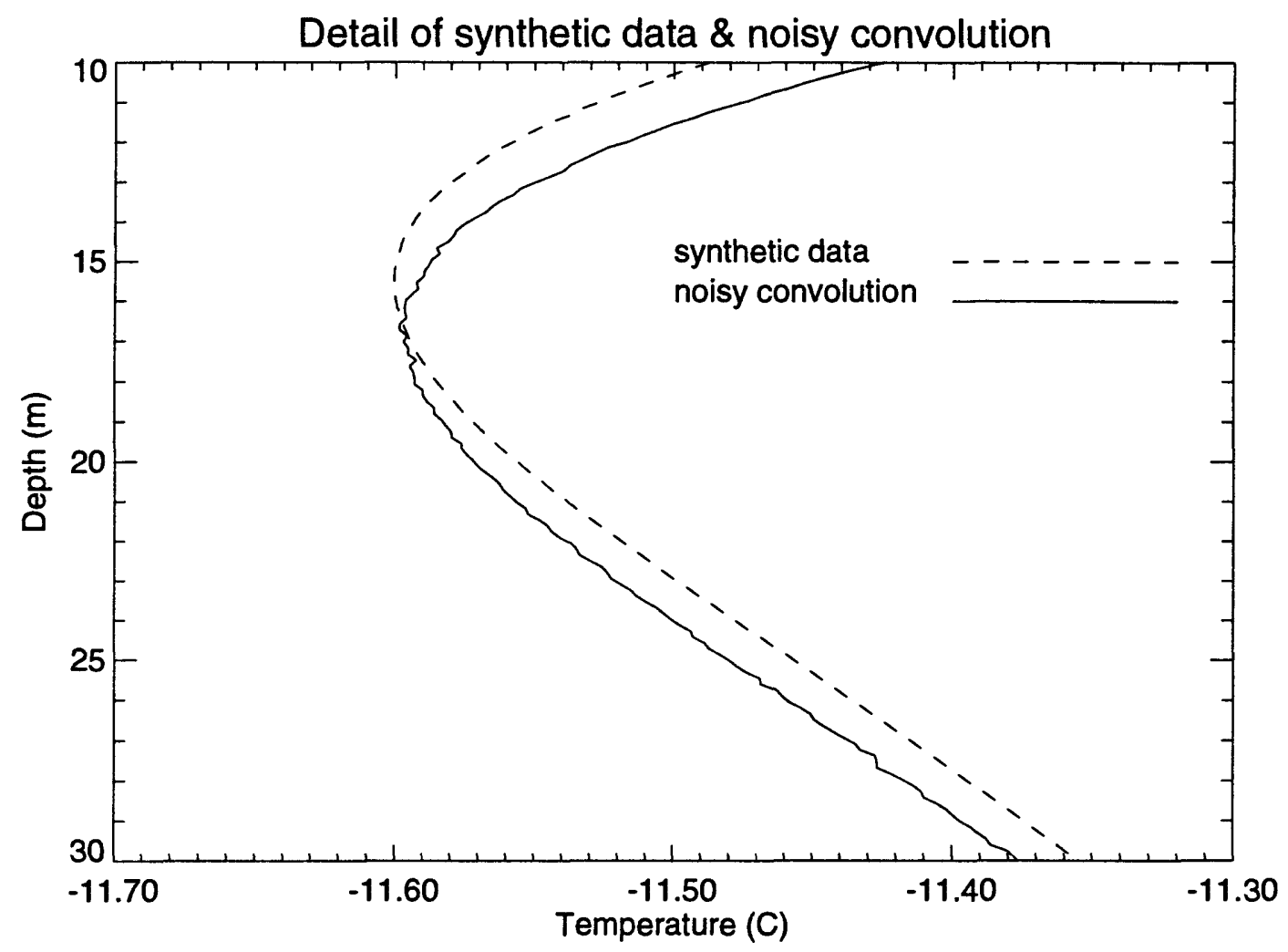

Figure 2: Detail plot of synthetic data (upper curve) and convolved data (lower curve) with $1 \mathrm{mK}$ random noise added. The depth of the minimum temperature is shifted down the borehole by about $2 \mathrm{~m}$.

An exponential probe response function was constructed using the computer code response.f (given in Appendix B) with $t_{o}=0, \tau=15 \mathrm{~s}$, a time interval of $0.05 \mathrm{~s}$, and a time cutoff of $100 \mathrm{~s}$. This probe response function was convolved with the synthetic data using the serial multiplication computer code convol.f (see Appendix B). The resulting signal shows the effect of the probe response (Figure 1).

To simulate data collected at $2 \mathrm{~s}$ intervals by a probe moving at $7.6 \mathrm{~cm} / \mathrm{s}(15 \mathrm{ft} /$ minute $)$, the convolved signal was then sampled at a $15 \mathrm{~cm}$ interval (every 40 th data point). Random (white) noise with a standard deviation of $1 \mathrm{mK}$ was added to this sampled signal. The resultant synthetic measurement (Figure 2) is representative of our expected data quality with the current measurement system (Clow and others, 1995).

The power spectrum for the noisy convolved signal (Figure 3) shows that the data signal departs significantly from the noise only for frequencies less than about $0.02 \mathrm{~Hz}(50$ second period, or $3.75 \mathrm{~m}$ at $7.6 \mathrm{~cm} / \mathrm{s}$ ). We use an exponential function to estimate the smooth signal plus noise function (Figure 3). The parameters for the exponential signal plus noise function were selected (by hand) to produce a conservative signal estimate (i.e., the model curve is systematically lower in squared amplitude than the calculated power spectrum). We modeled the noise with a constant squared amplitude of $10^{-10}$ 


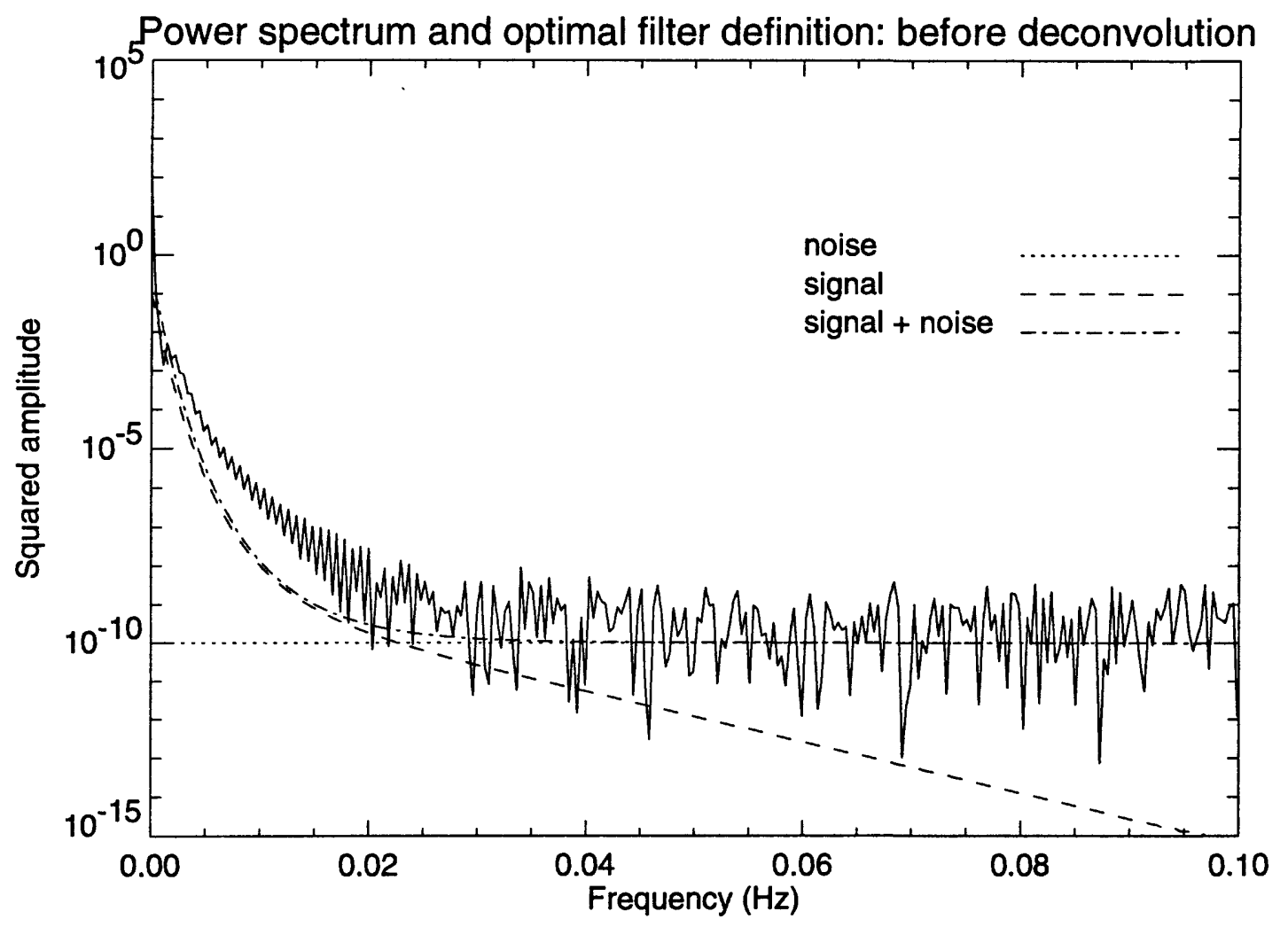

Figure 3: Power spectrum of the noisy convolution function based on the synthetic data set. The $1 \mathrm{mK}$ white noise spectrum is fit by a horizontal line at $10^{-10}$ squared amplitude. The noise plus signal function is fit by an exponential function of the form $d(f)=a+$ $b \exp (-c f)$ where $a=-10.0, b=+9.0$, and $c=150$. The modeled signal function is the difference of the two modeled functions.

for all frequencies. The difference between the modeled signal plus noise and and the modeled constant noise formed the estimate of the signal power spectrum for the optimal smoothing of the noisy convolved signal.

Table 2: Optimal filter functions - signal plus noise

\begin{tabular}{l|llllll}
\hline \multirow{2}{*}{$\begin{array}{l}\text { data } \\
\text { set }\end{array}$} & \multicolumn{4}{|c}{ Fitting function: $d(f)=a+b \exp (-c f)$} \\
before deconvolution & \multicolumn{2}{c}{ after deconvolution } \\
\hline synthetic & $a$ & $b$ & $c$ & $a$ & $b$ & $c$ \\
1992 GISP2 & -10 & 9 & 150 & -10 & 7 & 50 \\
& -10.9 & 10 & 1400 & -12 & 4 & 250
\end{tabular}

The smoothed result was then augmented by 9 points interpolated between each data point. This interpolated function was deconvolved with a discrete probe response function with an $0.2 \mathrm{~s}$ interval. The power spectrum of this deconvolution (Figure 4 ) shows that the 


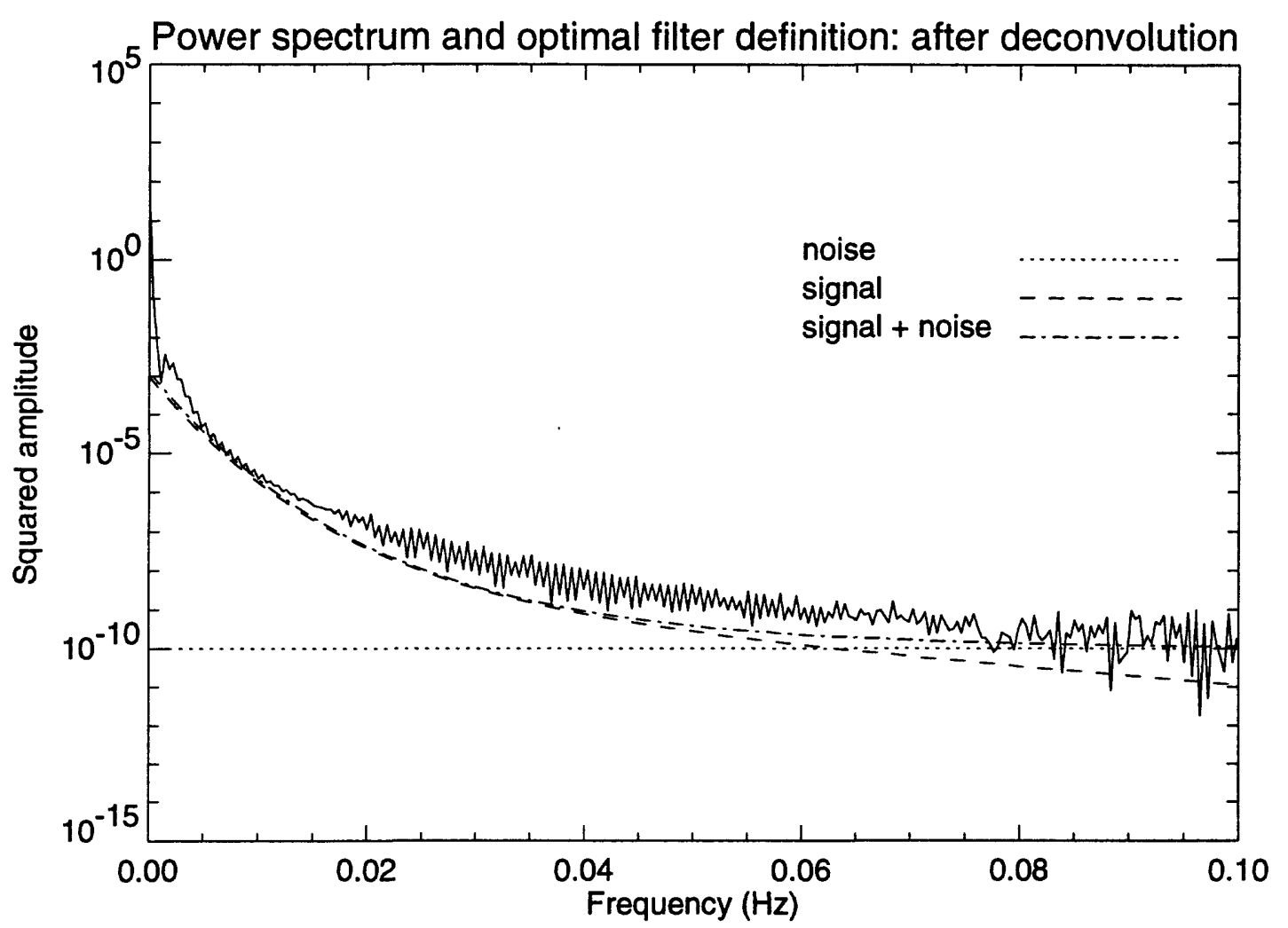

Figure 4: Power spectrum of the deconvolved signal based on the synthetic data set example. The white noise spectrum is fit by a horizontal line at $10^{-10}$ squared amplitude. The noise plus signal function is fit by an exponential function of the form $d(f)=a+$ $b \exp (-c f)$ where $a=-10.0, b=+7.0$, and $c=50$. The modeled signal function is the difference of the two modeled functions.

signal has been stretched to higher frequencies (i.e., the deconvolved signal has meaningful information to at least $0.04 \mathrm{~Hz}$ verses $0.02 \mathrm{~Hz}$ for the original data). Again a conservative exponential estimate was made of the signal function. A flat white noise function with a squared amplitude of $10^{-10}$ was again subtracted from the modeled signal plus noise to define the signal function. This optimal filter was then applied to smooth the deconvolved data.

The biggest potential problems in reconstruction of our synthetic data set are expected where the temperature gradients are largest. Between the depths of 14 and 18 meters (Figure 5) the synthetic data has a large variation in gradient. The recovered values agree closely with the original data.

Examination of difference histograms gives an idea of the accuracy of our analysis (Figure 6). The difference between the convolved data and the noisy convolved data has a standard deviation of $1 \mathrm{mK}$, by design (dotted line, Figure 6). After optimal smoothing, that difference falls to about $0.3 \mathrm{mK}$ (dashed line, Figure 6). The difference between our final, smoothed deconvolution and the original data function has a standard deviation of 
Detailed comparison of synthetic data, convolution, deconvolution

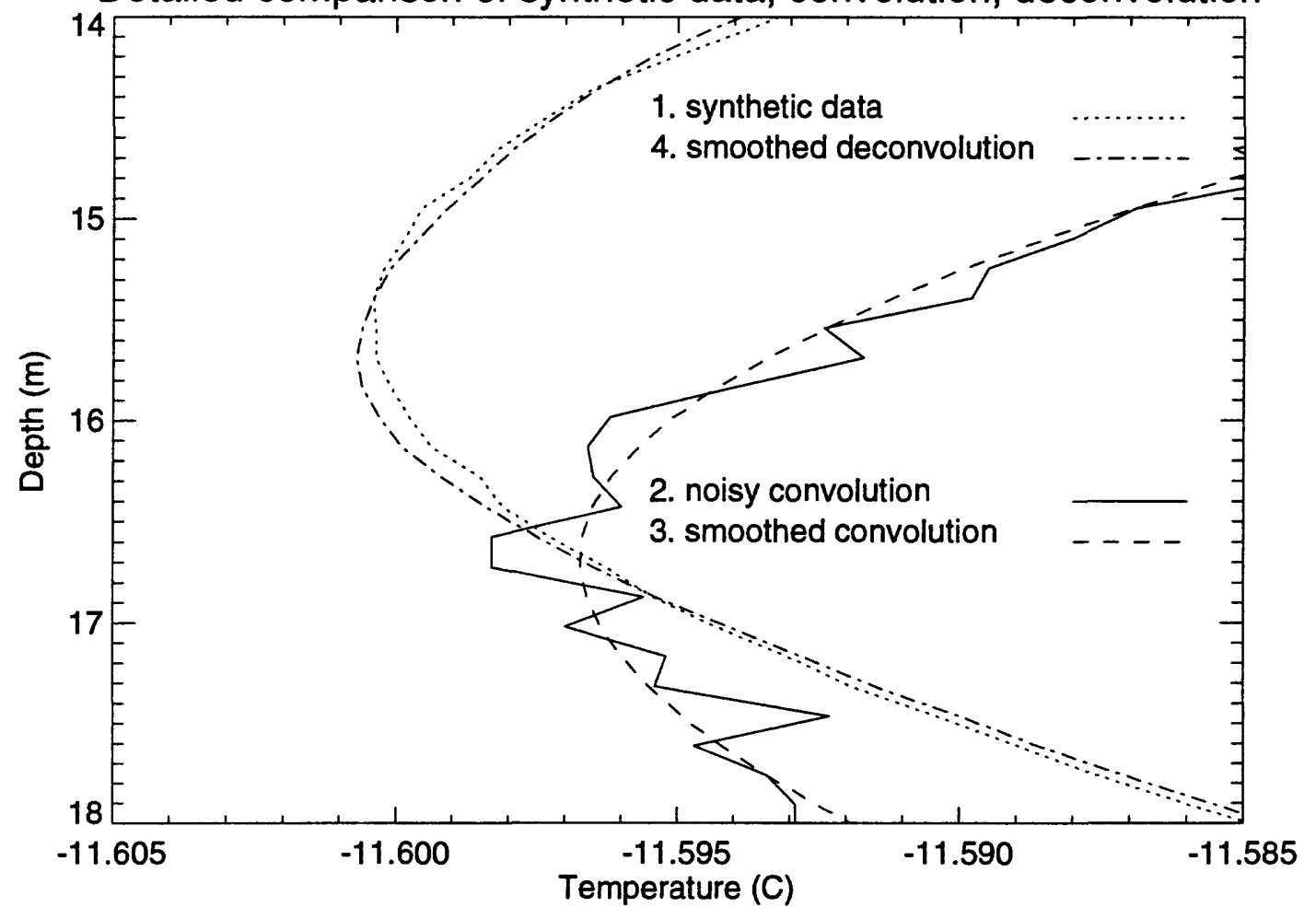

Figure 5: Detailed comparison of the synthetic data set, noisy convolution, optimally smoothed convolution, and smoothed deconvolution for the maximum curvature portion of the data set. 


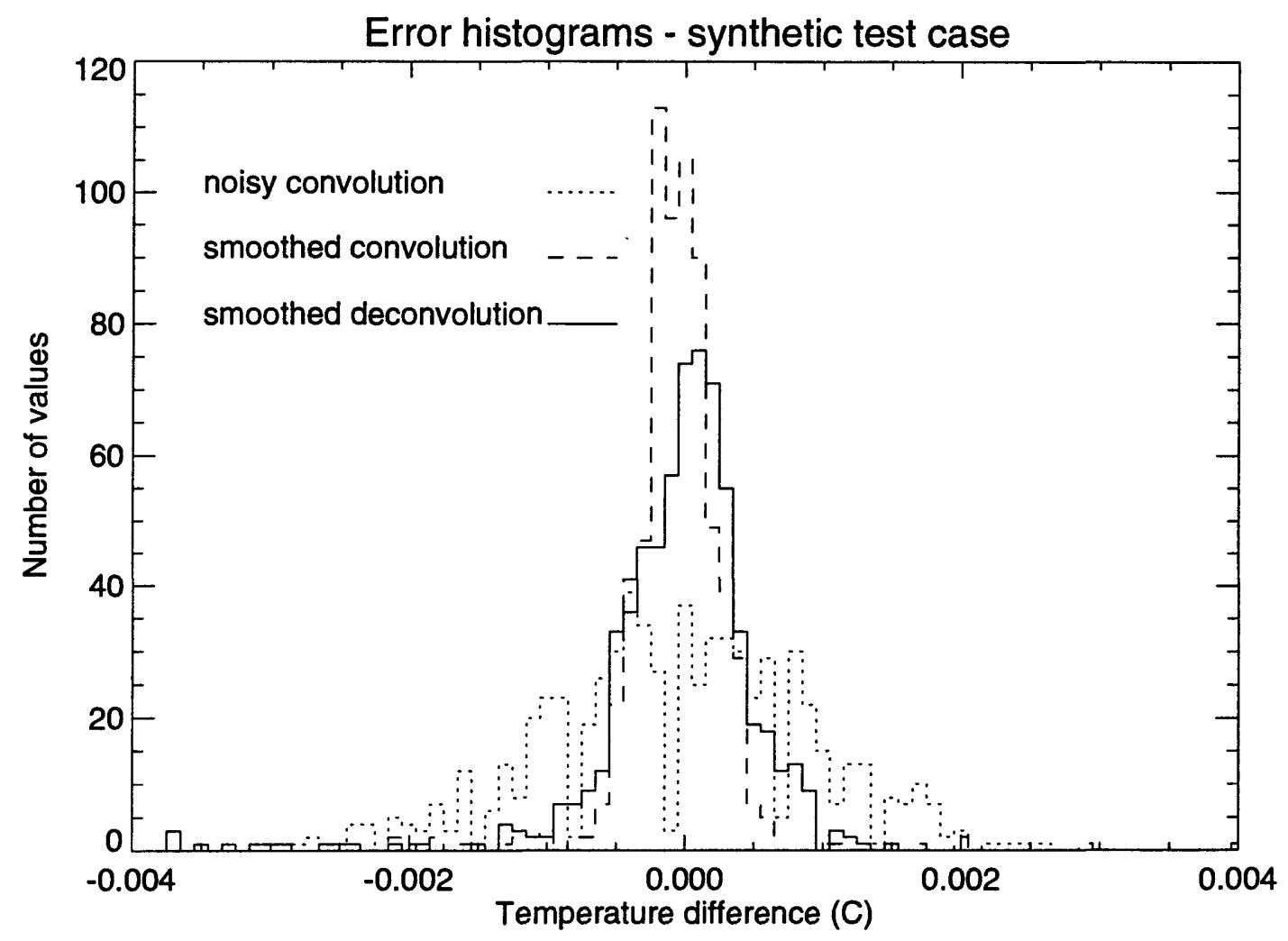

Figure 6: Histograms of differences between test data functions. Dotted line $=$ histogram of $1 \mathrm{mK}$ white noise added to convolved data function (difference between convolution and noisy convolution). Dashed line = histogram of convolution noise after optimal smoothing (difference between convolution and optimally smoothed noisy convolution). Solid line = histogram of total deconvolution error (difference between original synthetic data function and optimally smoothed deconvolution of smoothed noisy convolution).

less than $0.5 \mathrm{mK}$ (solid line, Figure 6).

\subsection{Deconvolution of measured temperatures in GISP2 borehole}

On 12 May 1992, before drilling operations began for the 1992 season, we obtained a temperature $\log$ in the GISP2 borehole in Greenland (Figure 7; Clow and others, 1995). This section discusses the preliminary noise filtering and deconvolution procedures applied to that data set. A three step procedure was used: 1 . removal of noise by optimal filtering, 2. deconvolution with an assumed probe response function, 3. optimal filtering for removal of deconvolution noise.

Examination of the power spectrum of the measured temperatures (Figure 8), shows that the flat, white-noise spectrum of the random slip-ring noise completely dominates to a frequency of about $0.004 \mathrm{~Hz}$. The spectrum is significantly affected by noise to at least $0.0025 \mathrm{~Hz}$. We chose a conservative exponential fit function (Figure 8); it severely damps the optimal filter for frequencies greater than $0.003 \mathrm{~Hz}$ (Figure 9 ). The difference 


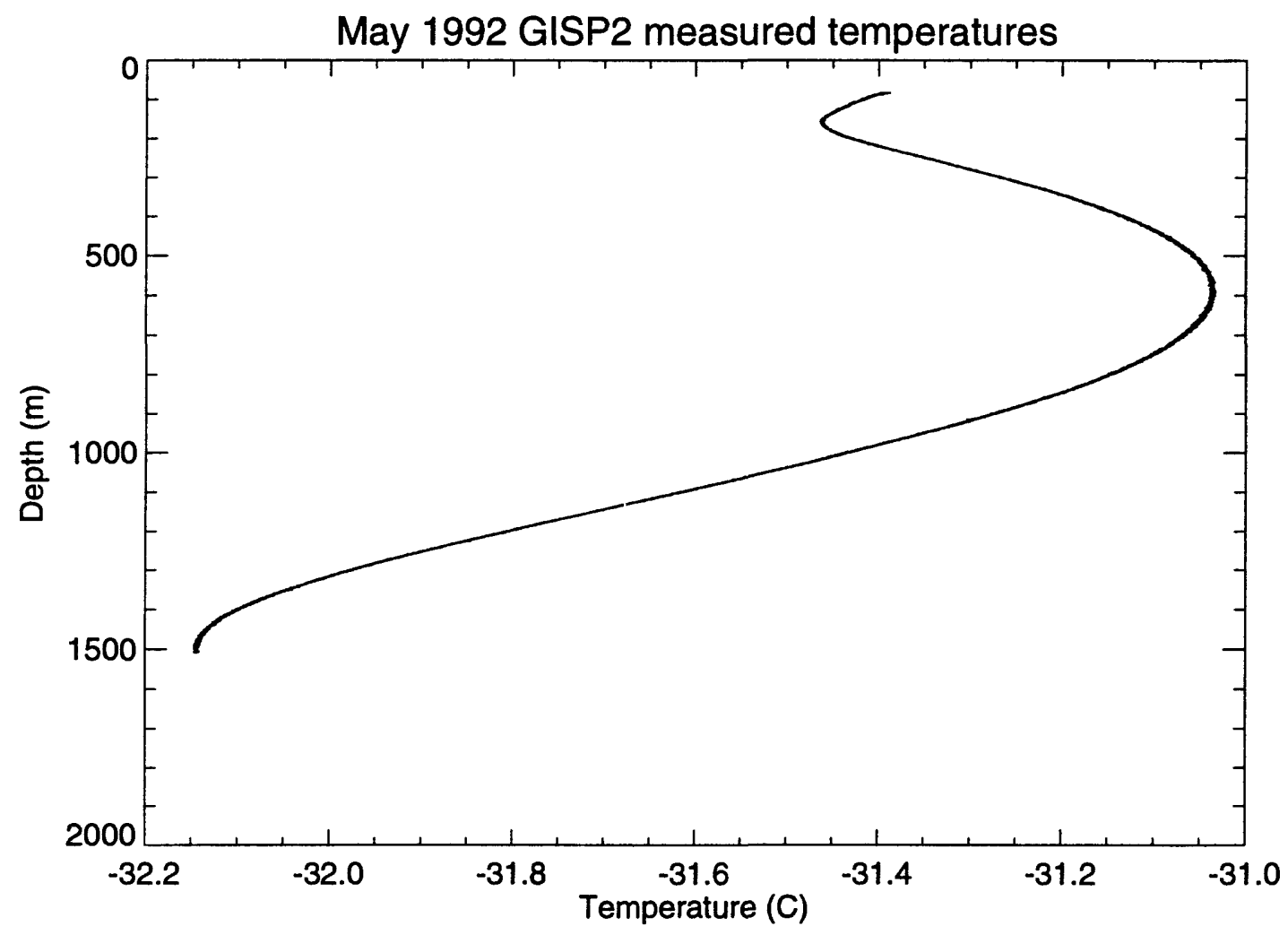

Figure 7: Measured GISP2 icecore hole temperature log of 12 May 1992. 


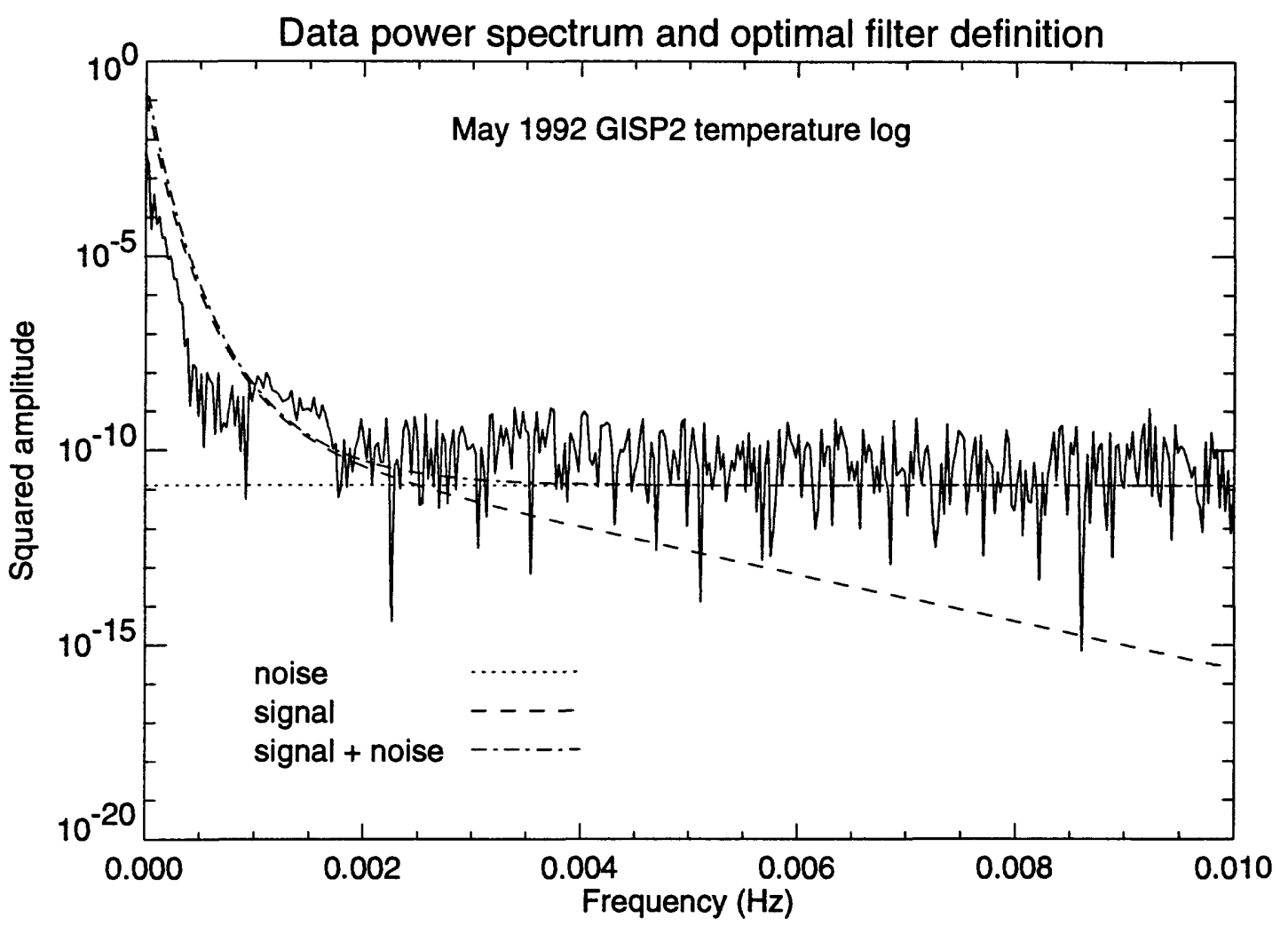

Figure 8: Power spectrum of GISP2 temperature log. The inferred white noise of the depth measurement system (so-called "slip-ring" noise) is modeled with a flat function at $10^{-10.9}$ squared amplitude. The noise plus signal function is fit by an exponential function of the form $d(f)=a+b \exp (-c f)$ where $a=-10.9, b=+10.0$, and $c=-1400$.

between the measured data and the optimally smoothed data follow a centrally peaked, approximately symmetric distribution with a standard deviation of about $1.5 \mathrm{mK}$ (Figure 10). This distribution agrees with our expectation from initial examination of the data (Clow and others, 1995). Compared to the power spectrum from the synthetic data example discussed in the last section, the noise in the GISP2 data set has about the same power, but persists to lower frequencies. The climate signal in the $80-1500 \mathrm{~m}$ portion GISP2 borehole is dominated by lower frequencies than our near-surface ( 0 - 100 m) synthetic example.

The smoothed data were deconvolved with an exponential discrete response function defined as follows: $t_{o}=0, \tau=15 \mathrm{~s}, \Delta t=2 \mathrm{~s}, t_{c}=100 \mathrm{~s}$. The function was generated by the FORTRAN code response.f (listed in Appendix B). The discrete response function was deconvolved with the smoothed data function by the serial division algorithm implemented in the FORTRAN code, simdecon.f (Appendix B).

The power spectrum of the deconvolved signal (Figure 11) shows that the deconvolution noise is several orders of magnitude lower in power than the original measurement noise. Again, the optimal filter (Figure 12) is designed as a conservative estimate for 


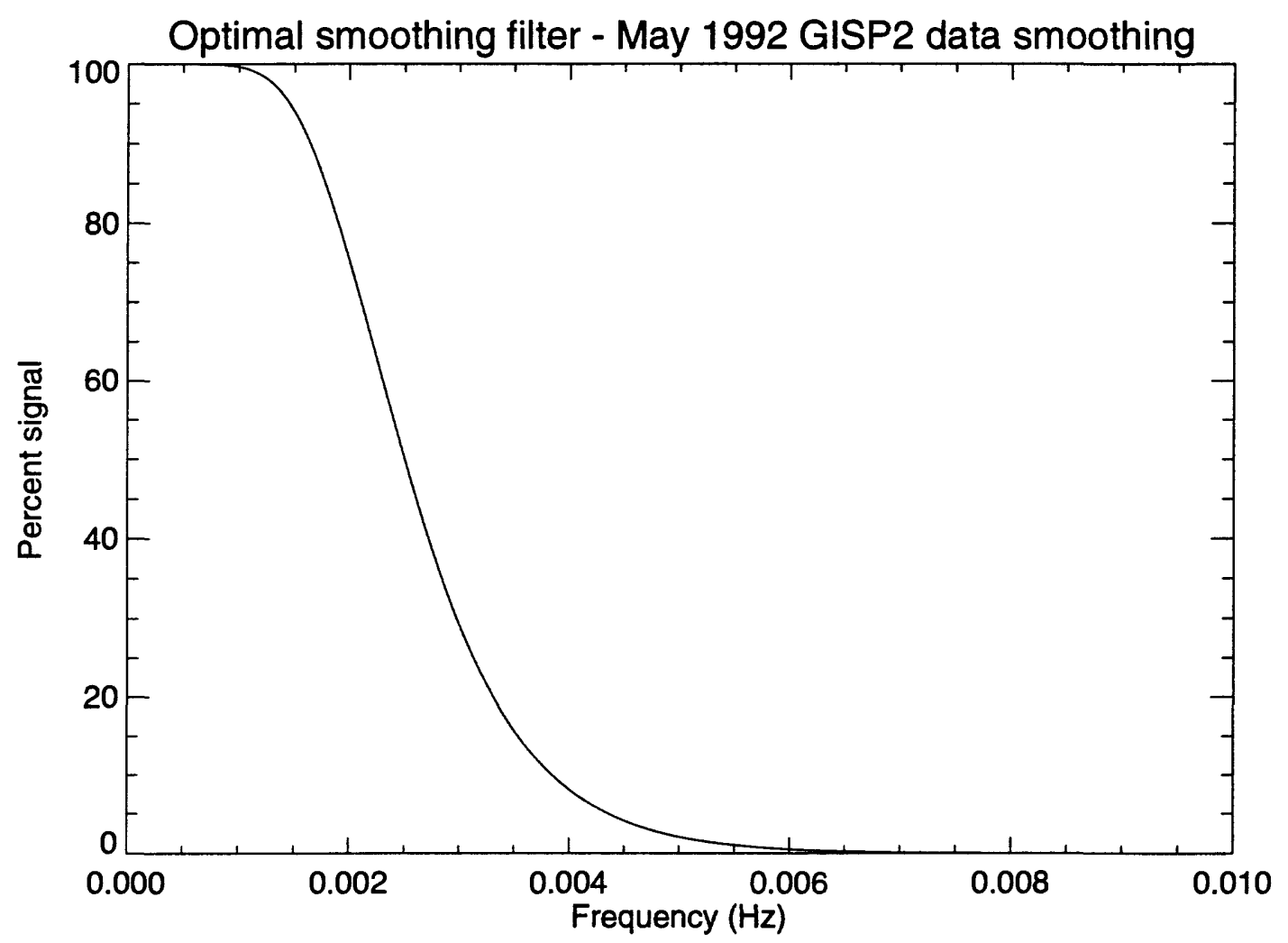

Figure 9: The optimal filter function, $\Phi$ used for smoothing of the GISP2 data log. 


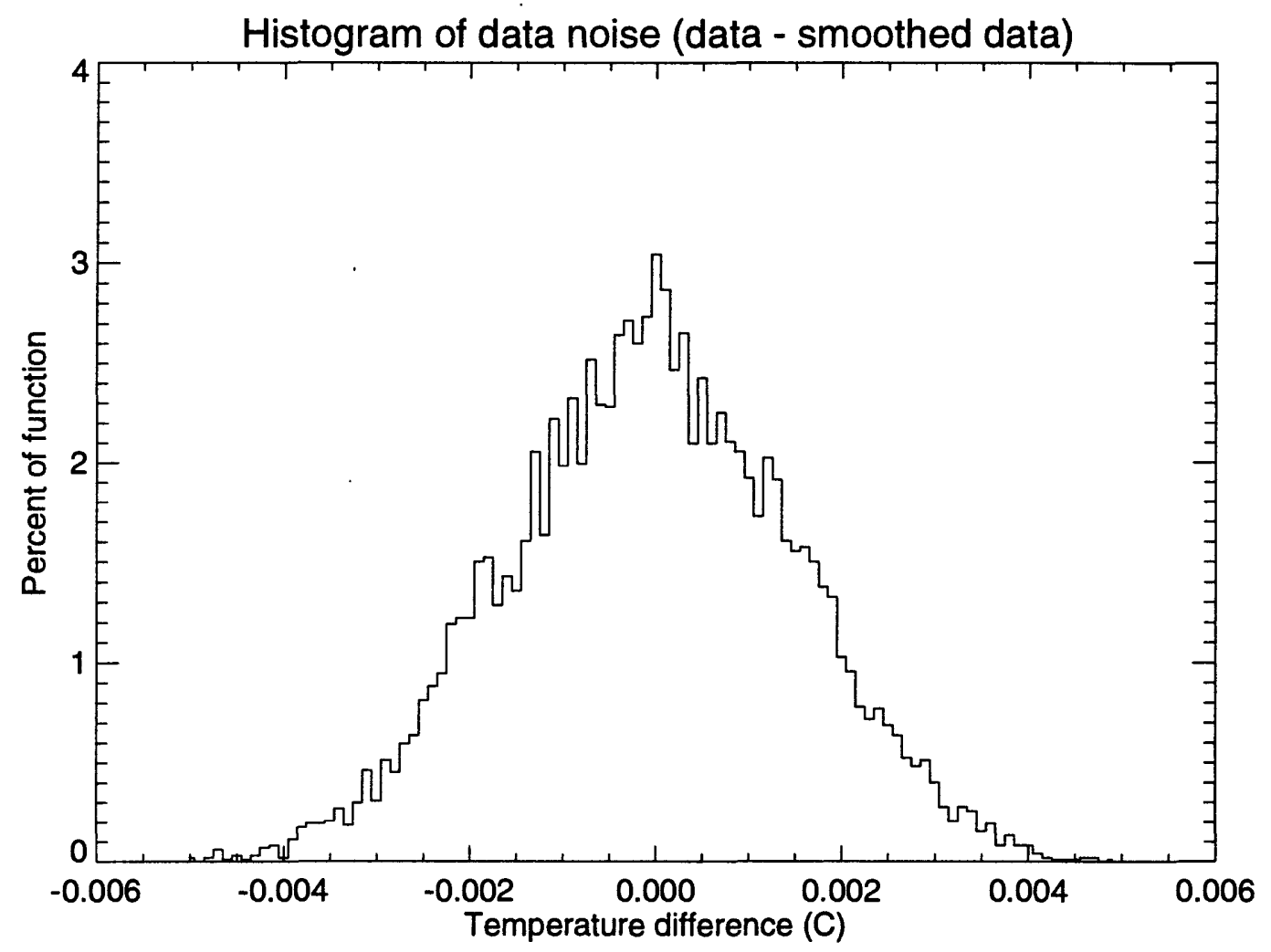

Figure 10: Histogram of difference between raw and smoothed temperature data from the 12 May 1992 GISP2 icecore hole. 


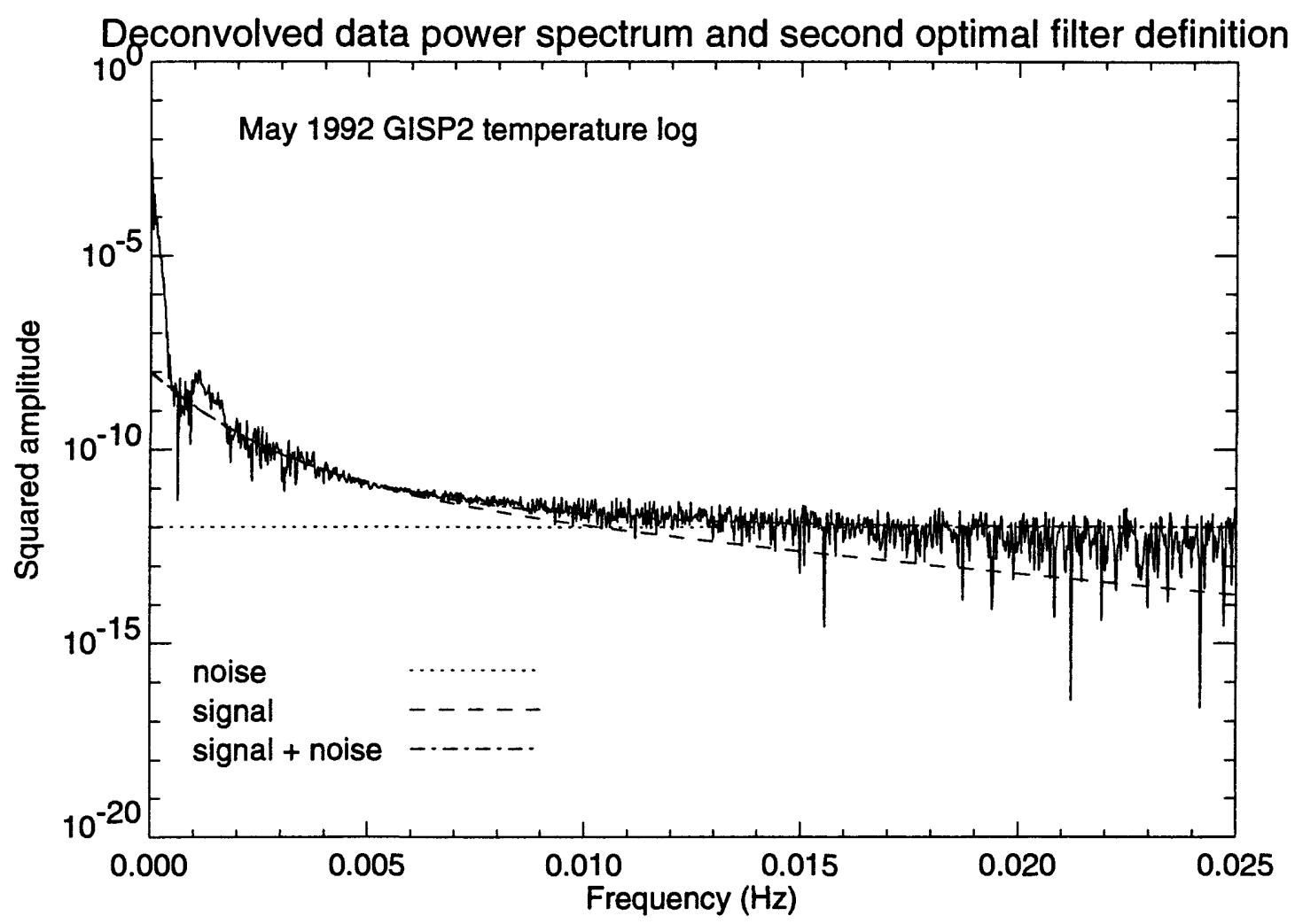

Figure 11: Power spectrum of the deconvolved GISP2 temperature log. The inferred white noise (numeric noise from discrete deconvolution) is modeled with a flat function at $10^{-12}$ squared amplitude. The noise plus signal function is fit by an exponential function of the form $d(f)=a+b \exp (-c f) ; a=-12.0, b=+4.0$, and $c=250$.

recovering a noise-free signal.

Examination of detailed subsections of the temperature data (Figure 13) shows the character of the measured data, smoothed data, and deconvolved signal. The difference between the smoothed data and the deconvolved signal (Figure 14) ranges from about -2 to $+2 \mathrm{mK}$ over the full depth of the temperature log. The positions of critical points in the temperature $\log$ (positions of gradient reversal, for example) are only shifted by a few meters by deconvolution (e.g., Figure 13b).

\section{CONCLUSION}

We have discussed a treatment of the deconvolution of borehole temperature measurements made at discrete time intervals by a finite-response probe system moving continuously in a borehole. High fidelity reconstruction of the actual temperature field from the measured temperatures requires careful attention to noise analysis and deconvolution. It is also important to consider the resolution of the discrete probe response function, particularly when dealing with high temperature gradients. Our synthetic example demonstrates 


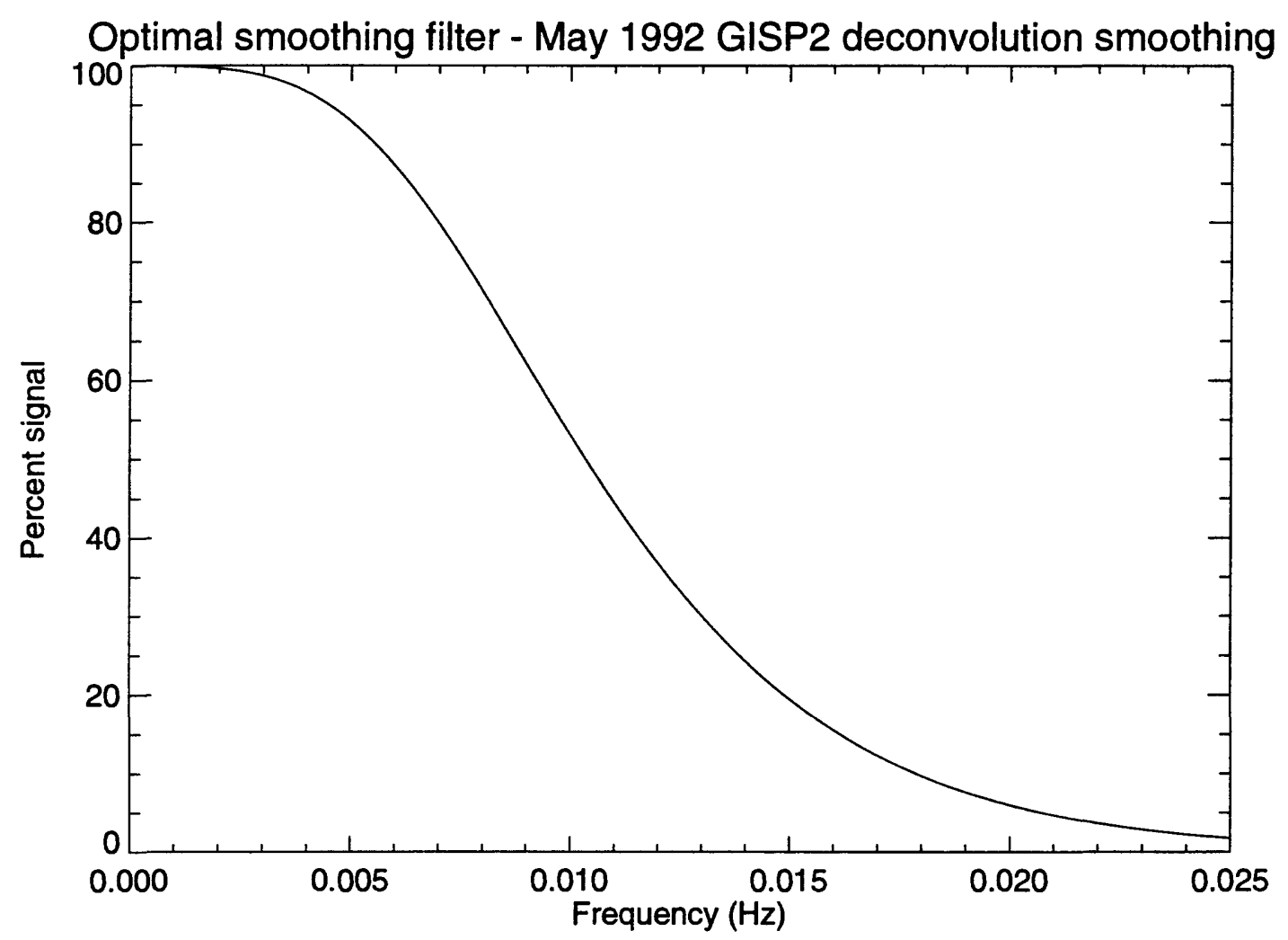

Figure 12: The optimal filter function, $\Phi$ used for smoothing of the deconvolution of the GISP2 data log. 

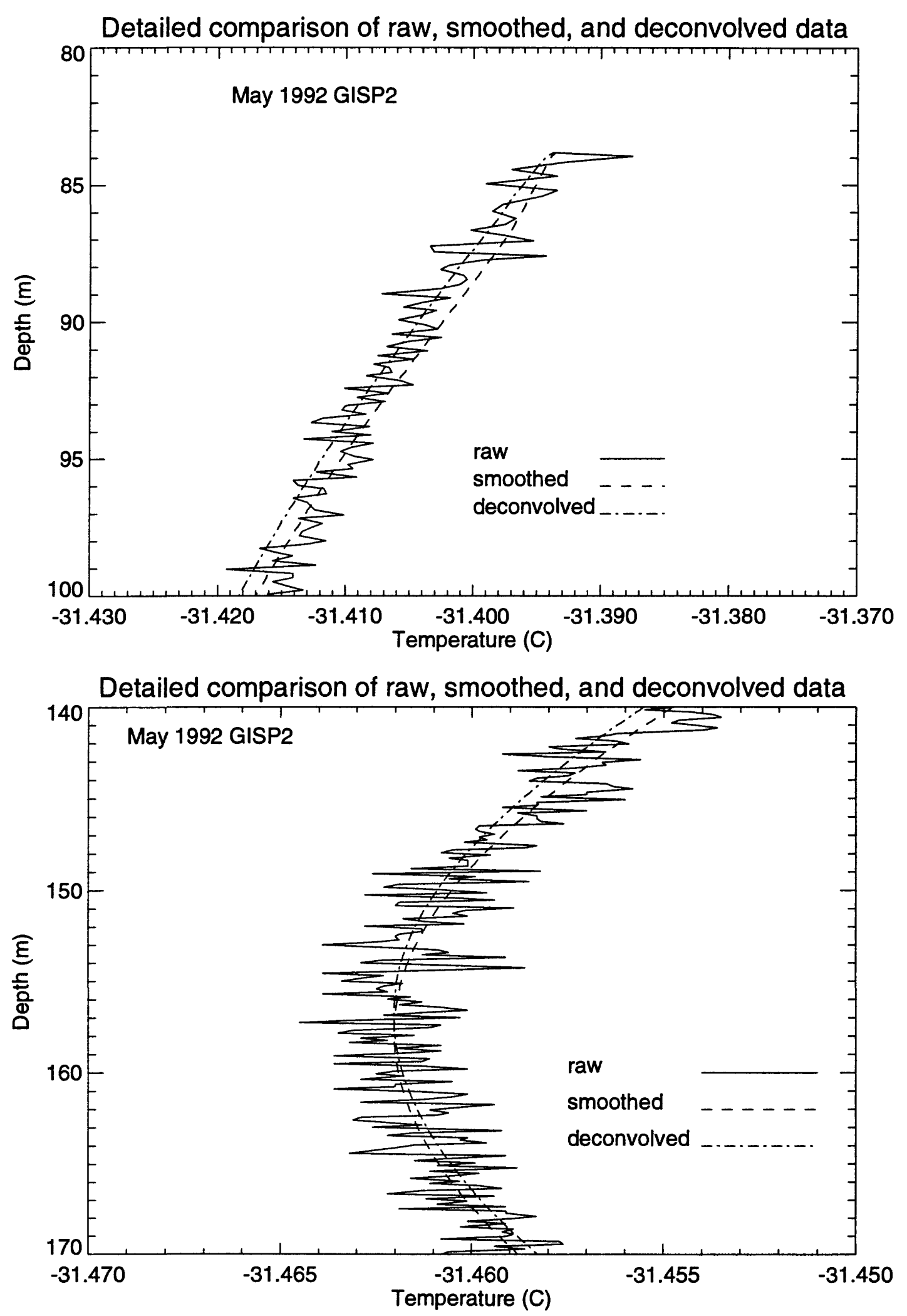

Figure 13: Detailed comparisons of measured data (noisy solid line), smoothed data (dashed line), and smoothed deconvolution (smooth solid line) for the GISP2 data set. 13a - top Top of $n$-butyl acetate fluid in the hole. 13b - bottom Region of maximum curvature. 

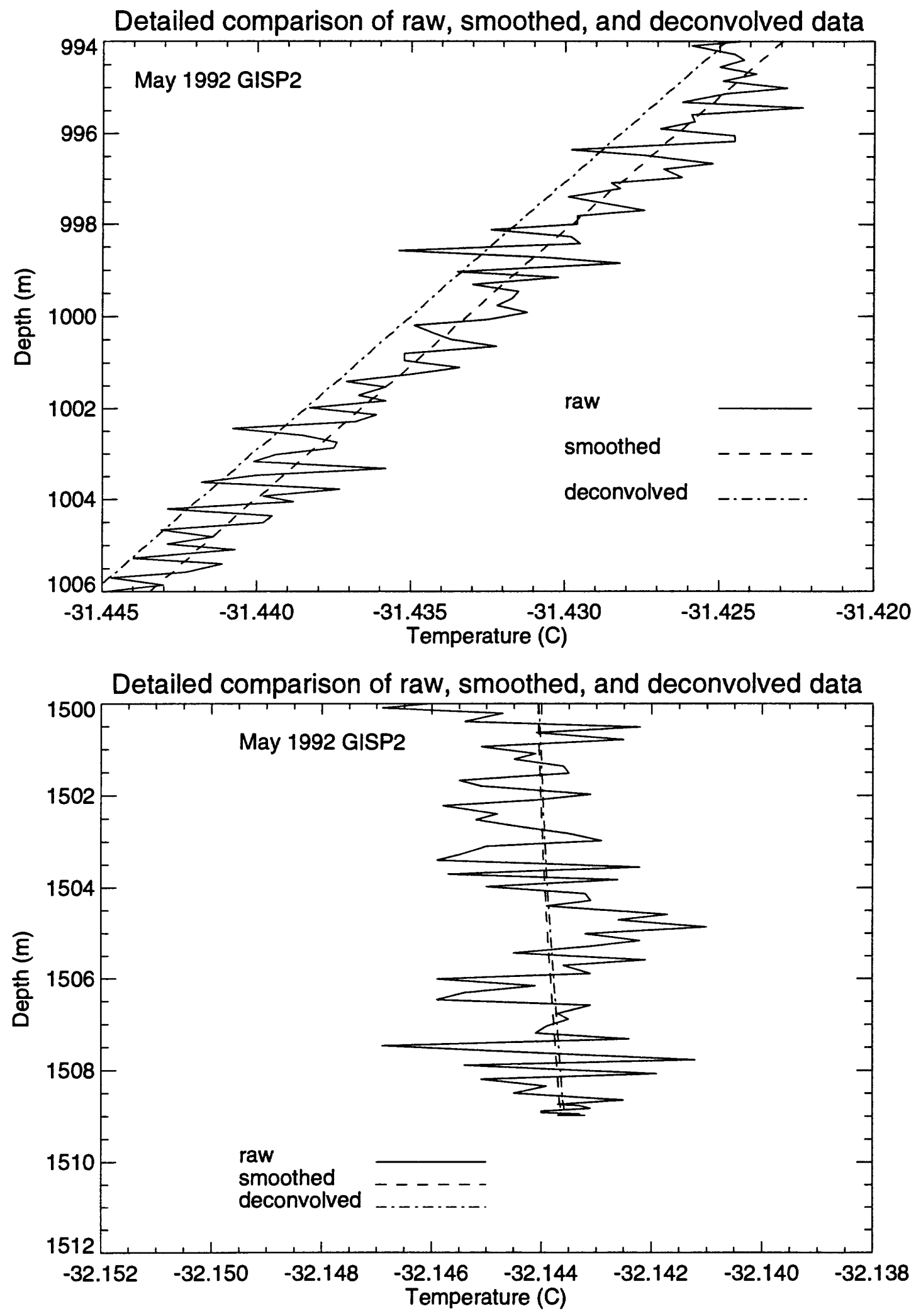

Figure 13: Detailed comparisons of measured data (noisy solid line), smoothed data (dashed line), and smoothed deconvolution (smooth solid line) for the GISP2 data set. 13c - top Region of maximum slope. 13d - bottom Bottom of the hole. 


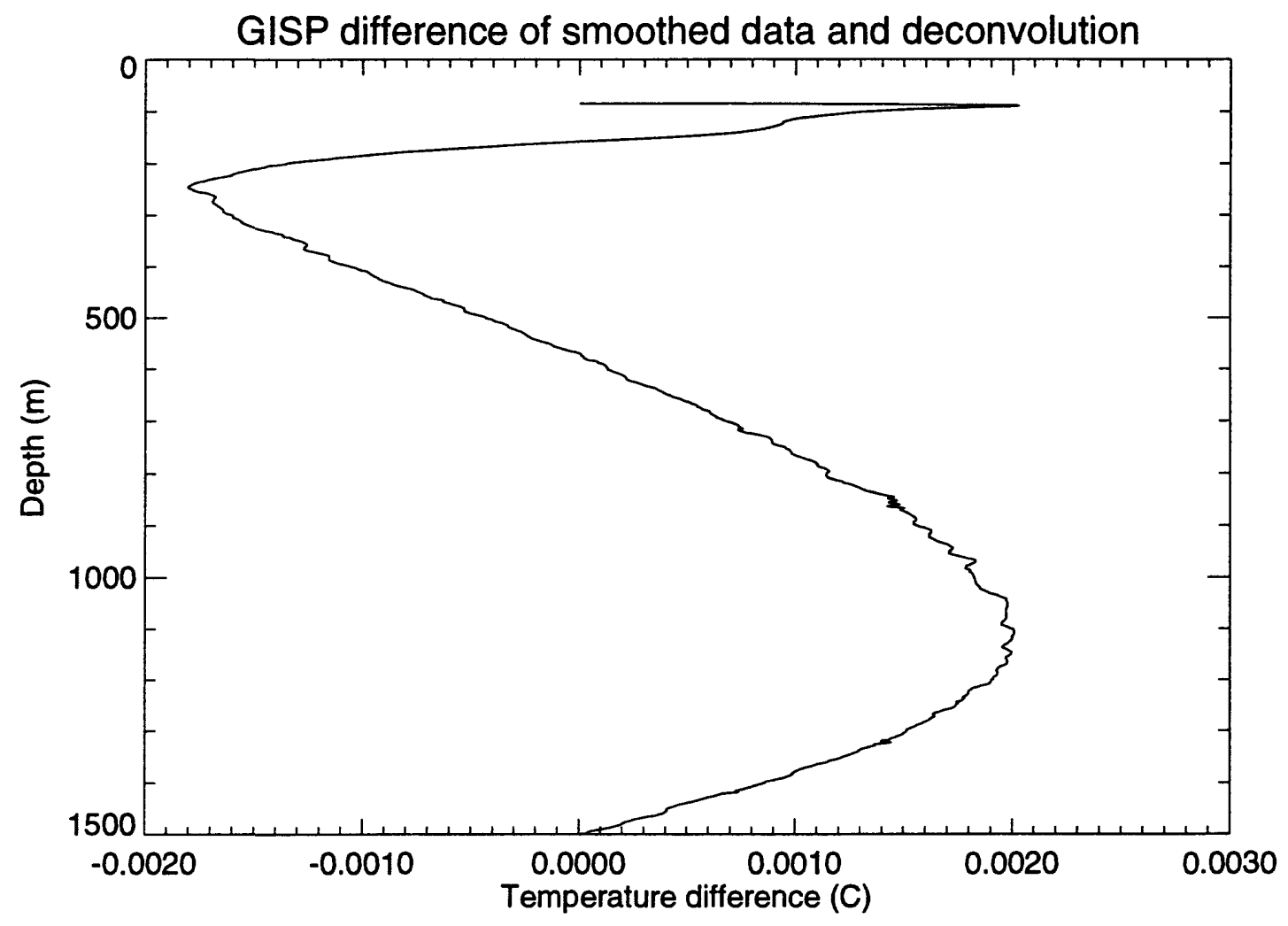

Figure 14: Difference between smoothed data and deconvolved data for the May 1992 GISP2 temperature log. 
that actual temperatures can be recovered to better than $1 \mathrm{mK}$ in the presence of noise of that magnitude. We applied a three-step method (optimal noise filtering of the measured data, discrete deconvolution with a measured probe response function, and optimal noise filtering of the deconvolution noise) to temperature data collected in May 1992 at the GISP2 borehole in Greenland. Examination of the power spectrum from the GISP2 data set indicates that the $1.5 \mathrm{mK}$ random, slip-ring noise dominates to frequencies of $0.004 \mathrm{~Hz}$ and above. The deconvolved temperatures differ from the smoothed temperature measurements by less than $2 \mathrm{mK}$. Positions of key features in the temperature $\log$ (such as points of maximum gradient), are shifted by several meters as a result of deconvolution.

\section{ACKNOWLEDGEMENTS}

We thank Jack Kennelly for his work on design and construction of the high-precision logging system. Jeff Phillips gave us guidance on optimal filtering in the Fourier domain. Careful reviews by Karl Ellefsen and Mark Gettings improved this report. 


\section{References}

Beck, A.E., and Balling, N., 1988, Determination of virgin rock temperatures, in Haenel, R., Ryback, L., and Stegena, L., eds., Handbook of Heat-Flow Density Determination: Klewer Academic Publishers, p. 59-85.

Birch, F., 1948, The effects of Pleistocene climatic variations upon geothermal gradients: Americal Journal of Science, v. 246, p. 729-760.

Boulton, G.S., 1993, Greenland ice sheet; two cores are better than one: Nature, v. 366, p. $507-508$.

Bracewell, R.N., 1986, The Fourier Transform and Its Applications: New York, McGrawHill Book Company, 474 p.

Chisholm, T.J., and Chapman, D.S., 1992, Climate change inferred from analysis of horehole temperatures; an example from western Utah: Journal of Geophysical Research, v. 97, p. 14,155-14,175.

Clow, G.D., Saltus, R.W., and Waddington, E.D., 1995, High-precision temperature logging at GISP2, Greenland, May 1992: U.S. Geological Survey Open-file report (in press),

Clow, G.D., 1992, The extent of temporal smearing in surface-temperature histories derived from borehole temperature measurements: Palaeogeography, Palaeoclimatology, Palaeoecology, v. 98, p. 2157-2160.

Conaway, J.G., 1977, Deconvolution of temperature gradient logs: Geophysics, v. 42, p. 823-837.

Conaway, J.G., and Beck, A.E., 1977, Continuous logging of temperature gradients: Tectonophysics, v. 41, p. 1-7.

Costain, J.K., 1970, Probe response and continuous temperature measurements: Journal of Geophysical Research, v. 75, p. 3969-3975.

Lachenbruch, A.H., 1994, Permafrost, the active layer, and changing climate: U.S. Geological Survey Open-file report 94-694, $43 \mathrm{pp}$.

Lachenbruch, A.H., Cladouhos, T.T., and Saltus, R.W., 1988, Permafrost temperature and the changing climate: Permafrost, Fifth International Conference on Permafrost: Trondheim, Norway, Tapir Publishers, v. 3, p. 9-17.

Lachenbruch, A.H., and Marshall, B.V., 1986, Changing climate: Geothermal evidence from permafrost in the Alaskan Arctic: Science, v. 234, p. 689-696.

Morrison, M., 1994, GISP2; Earth's climate system revealed: Geotimes, v. 38, p. 20-22.

Nielsen, S.B., and Balling, N., 1984, Accuracy and resolution in continuous temperature logging: Tectonophysics, v. 103, p. 1-10. 
Press, W.H., Flannery, B.P., Teukolsky, S.A., and Vetterling, W.T., 1992, Numerical Recipes in FORTRAN: The Art of Scientific Computing, Second Edition: Cambridge, Cambridge University Press, $963 \mathrm{p}$.

Research Systems, Inc., 1993, IDL Reference Guide: Interactive Data Language Version 3.1: Boulder, CO 80303, Research Systems, Inc., 491 p.

Sass, J.H., Lachenbruch, A.H., Munroe, R.J., Greene, G.W., and Moses, T.H., Jr., 1971, Heat flow in the western United States: Journal of Geophysical Research, v. 76, p. 6376-6413.

Sawyer, D.S., Bangs, N.L., and Golovchenko, X., 1994, Deconvolving ocean drilling program temperature logging tool data to improve borehole temperature estimates: Chile Triple Junction: Journal of Geophysical Research, v. 99, p. 11,995-12,003.

Thomson, W., 1862, On the secular cooling of the earth: Transactions of the Royal Society of Edinburgh, v. 23, p. 157-170. 


\section{APPENDIX A: MEASUREMENT OF PROBE RESPONSE FUNCTION}

The Fenwal K212E custom-made thermistor probe used in the USGS high-precision logging system consists of 20 small NTC bead thermistors divided into two groups, each sealed in glass (Sass and others, 1971; Clow and others, 1995). The thermistor packets are wired in parallel and contained in a $4.0 \mathrm{~mm}$ diameter stainless steel needle. Chisholm and Chapman (1992) report a $7 \mathrm{~s}$ time constant for their Fenwal 212E temperature probe in still water. At cold temperatures and in other liquids we have measured significantly longer response times.

As discussed previously, equation (1) can be used to fit the response of a thermistor probe to a step change in temperature and equation (2) allows us to graphically determine the probe response from measurement of a step change in temperature. We have only conducted limited experimental tests of probe response so far.

For the Fenwal K212E probe in stirred (Figure A1) and unstirred alcohol (Figure A2), still water (Figure A3), and still Jet A Fuel (Figure A4) we measured response times ranging from 2.7 to $14.7 \mathrm{~s}$ (see Table 1). For these tests the probe started at ambient air temperature (about $29^{\circ} \mathrm{C}$ ) and was then submerged in a temperature bath at about 13 ${ }^{\circ} \mathrm{C}$ (alcohol and Jet A) or $0^{\circ} \mathrm{C}$ (water/ice bath). It is interesting to note that equation (1) is evidently not a good model for the later parts of the approach to equilibrium (the slope of the curve on the semi-log plot increases with time). Further tests of probe response are necessary to develop a better response function.

At the GISP2 icecore hole in Greenland we made an estimate of the probe response time by recording the probe's approach to an equilibrium temperature when first entering the liquid $n$-butyl acetate near the top of the hole (fluid first encountered at about $80 \mathrm{~m}$ depth). The probe was driven into the hole at $6 \mathrm{~cm} / \mathrm{s}(12 \mathrm{ft} / \mathrm{min})$ and stopped once the entire probe and weight assembly was immersed. Temperature measurements were made at $5 \mathrm{~s}$ intervals (Figure A5) as the probe and weight assembly reached thermal equilibrium. Based on the probe response from 18:05:45 to 18:06:40 (Figure A6), a response time of $15 \mathrm{~s}$ (Figure A6) can be derived. This is a preliminary determination for the probe response in the physical conditions encountered at the GISP2 borehole. Further measurements must be made in subsequent field seasons and in the laboratory to improve our understanding of the probe response. 
Probe System Response in Stirred Alcohol

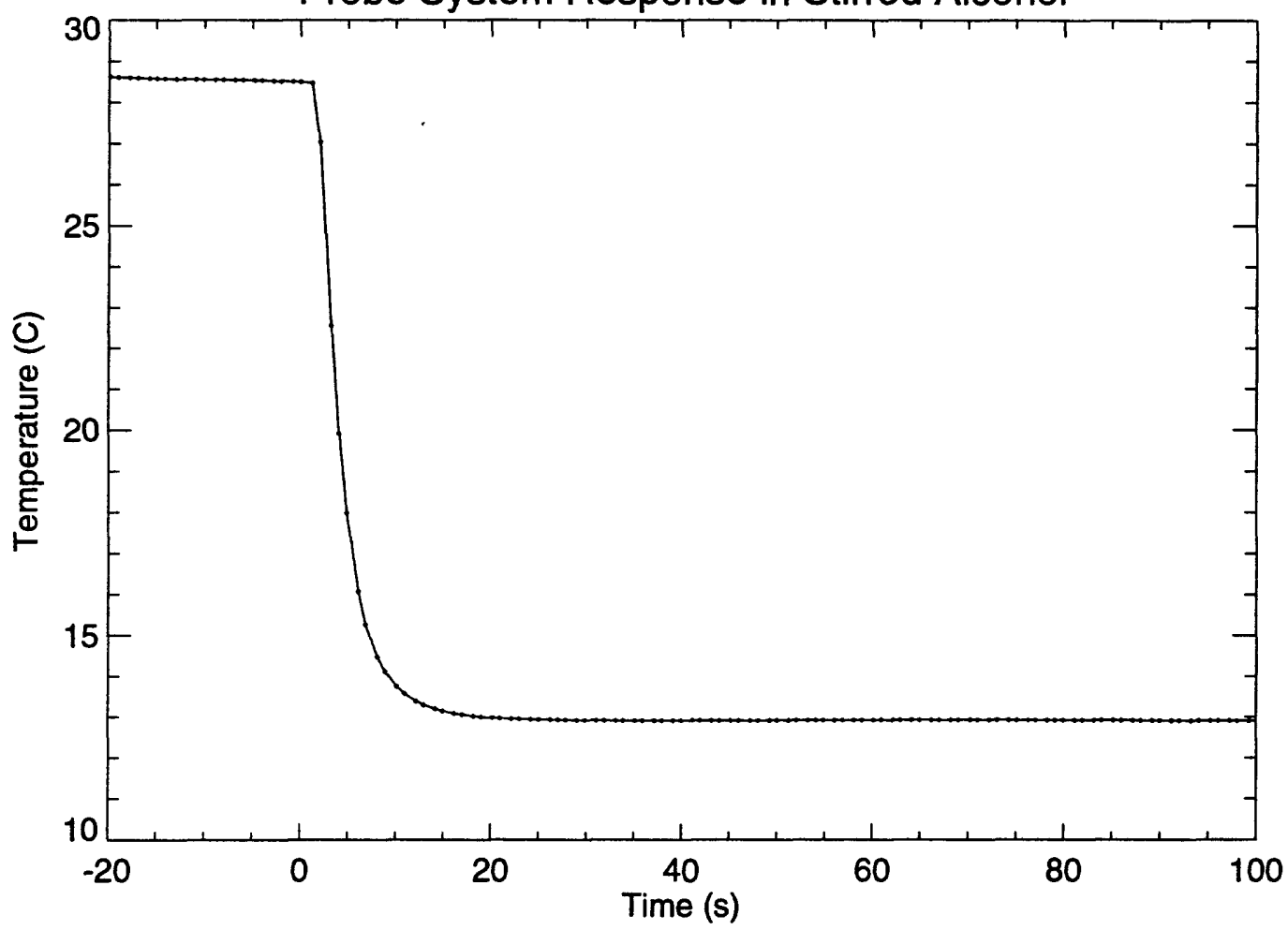

Time Constant in Stirred Alcohol

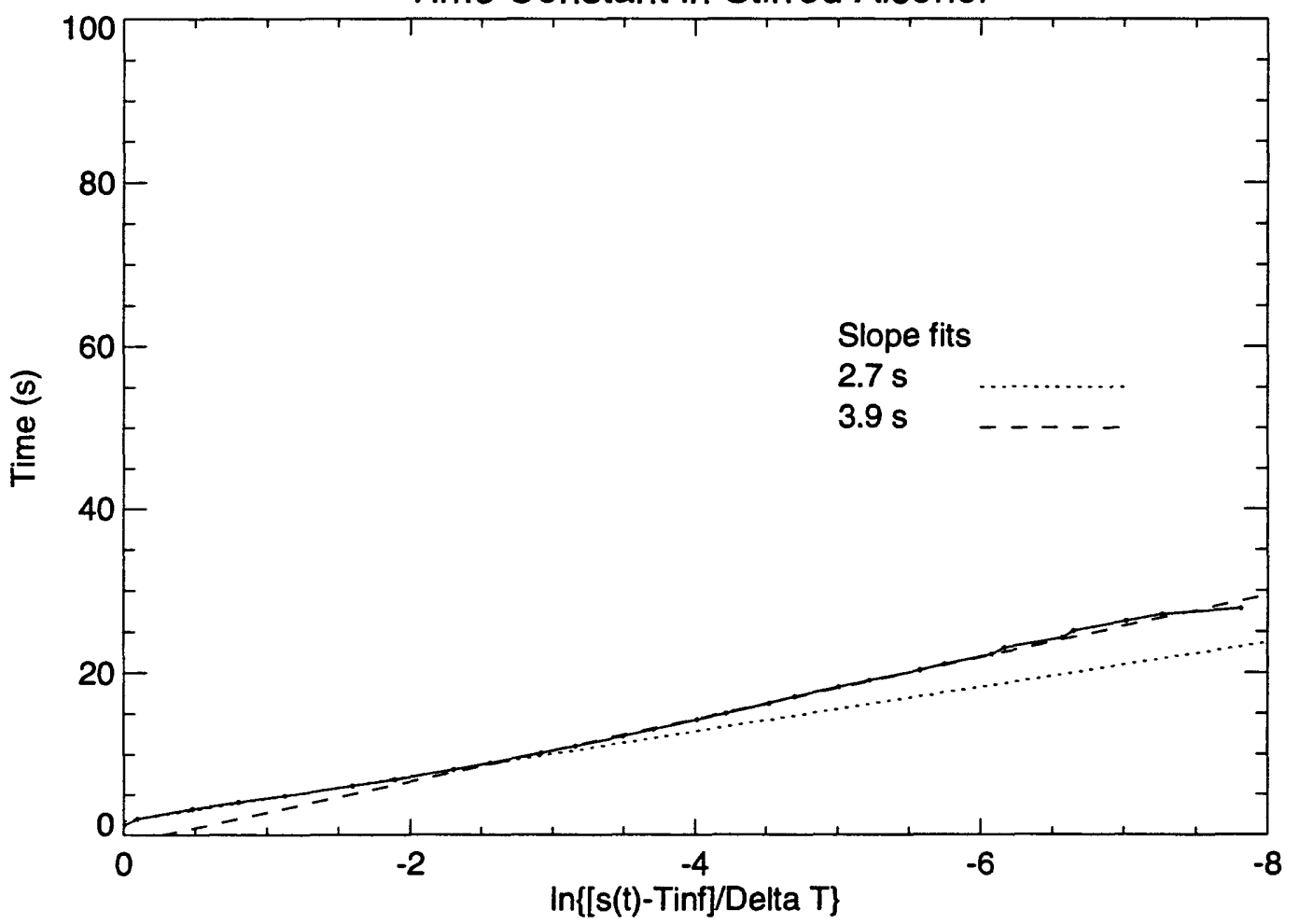

Figure A1: (top) Temperature verses time plot for Fenwal 212E thermister probe immersion in stirred alcohol at $13^{\circ} \mathrm{C}$. (bottom) Time verses $\ln \left[\left(s(t)-T_{\infty}\right) / \Delta T\right]$ plot of Fenwal $212 \mathrm{E}$ thermister probe response in stirred alcohol at $13{ }^{\circ} \mathrm{C}$. Initial time constant $=2.7 \mathrm{~s}$. 


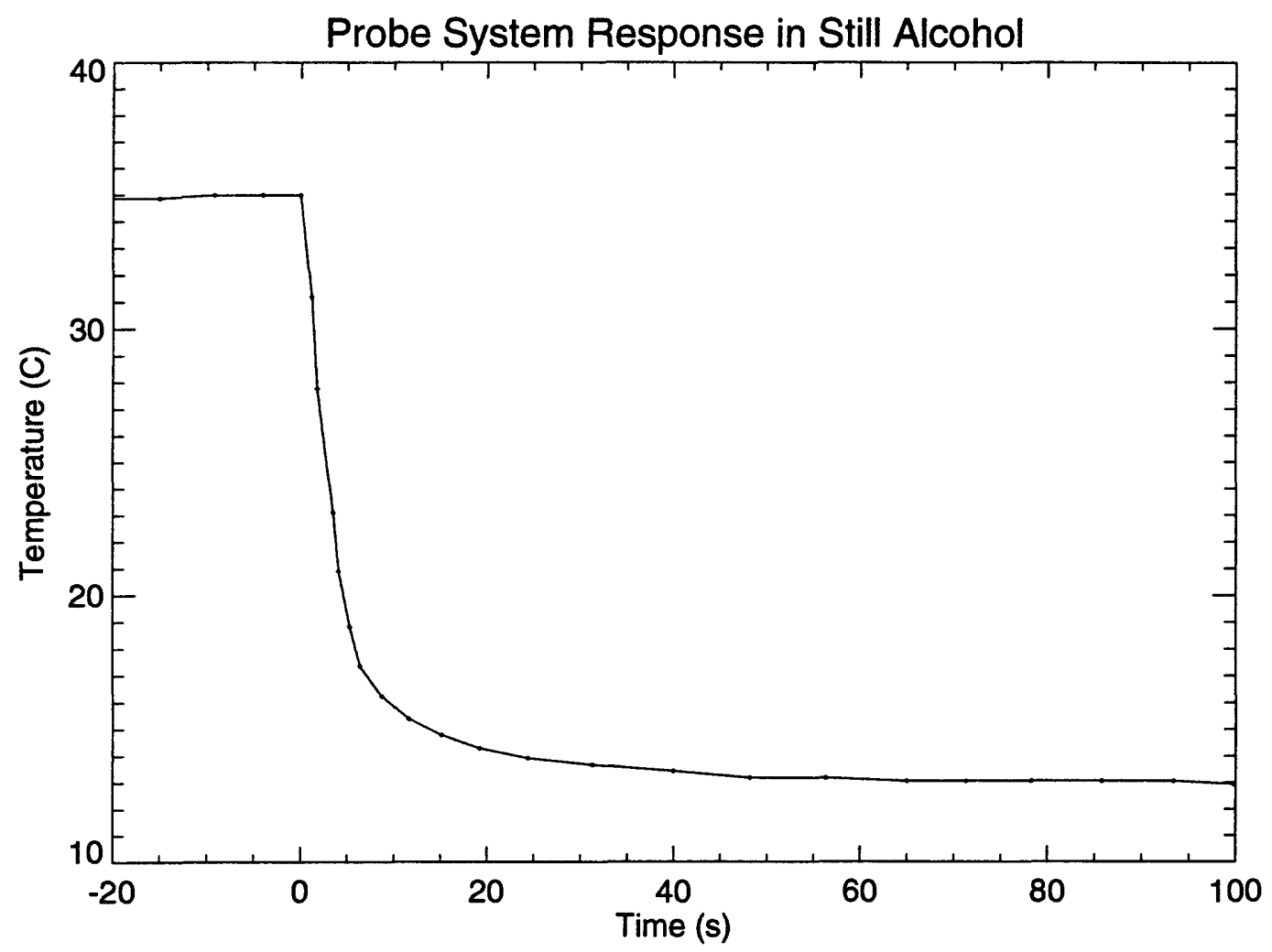

Time Constant in Still Alcohol

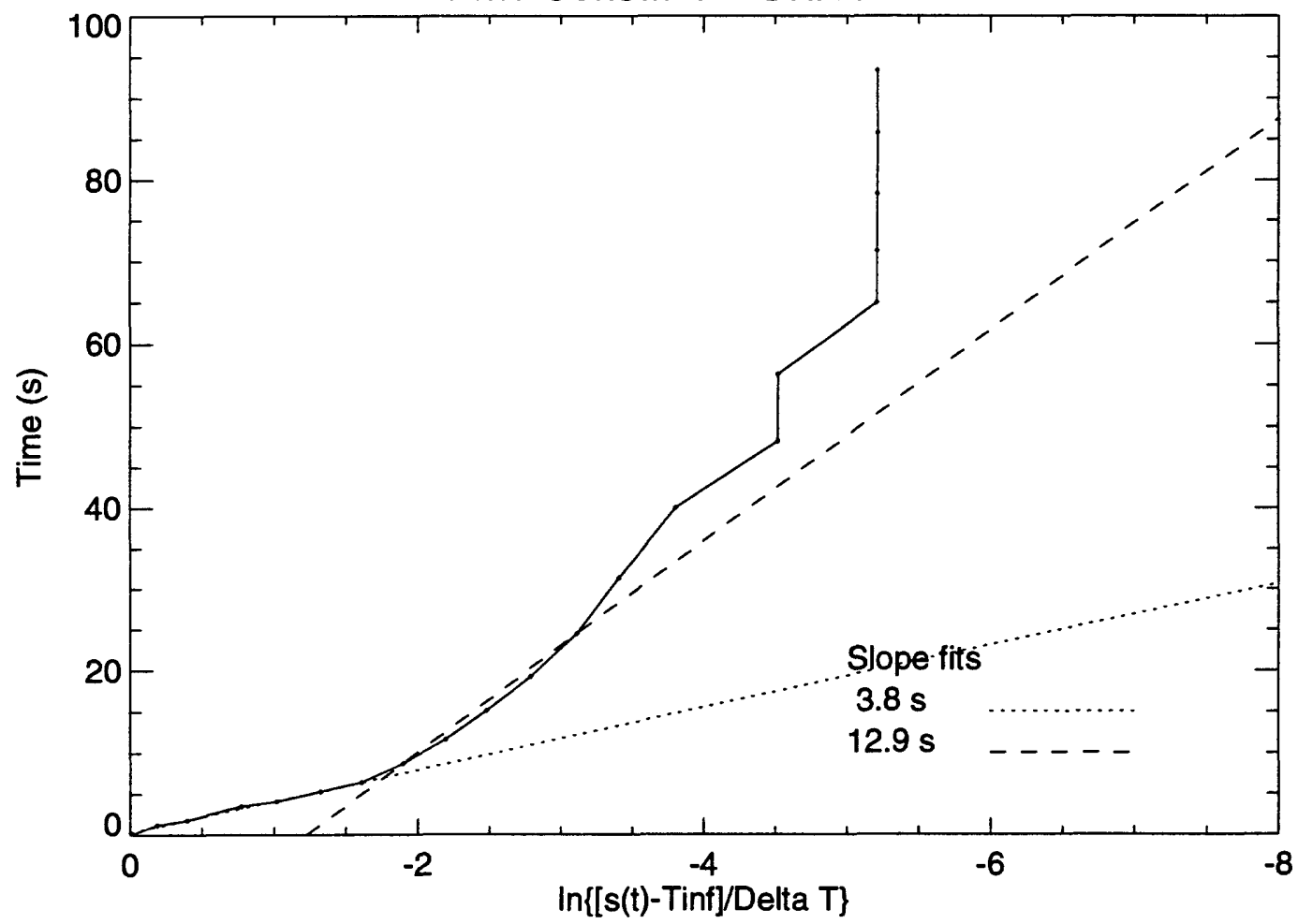

Figure A2: (top) Temperature verses time plot for Fenwal 212E thermister probe immersion in unstirred alcohol at $13^{\circ} \mathrm{C}$. (bottom) Time verses $\ln \left[\left(s(t)-T_{\infty}\right) / \Delta T\right]$ plot of Fenwal $212 \mathrm{E}$ thermister probe response in unstirred alcohol at $13{ }^{\circ} \mathrm{C}$. Time constant $=$ $3.8 \mathrm{~s}$. 


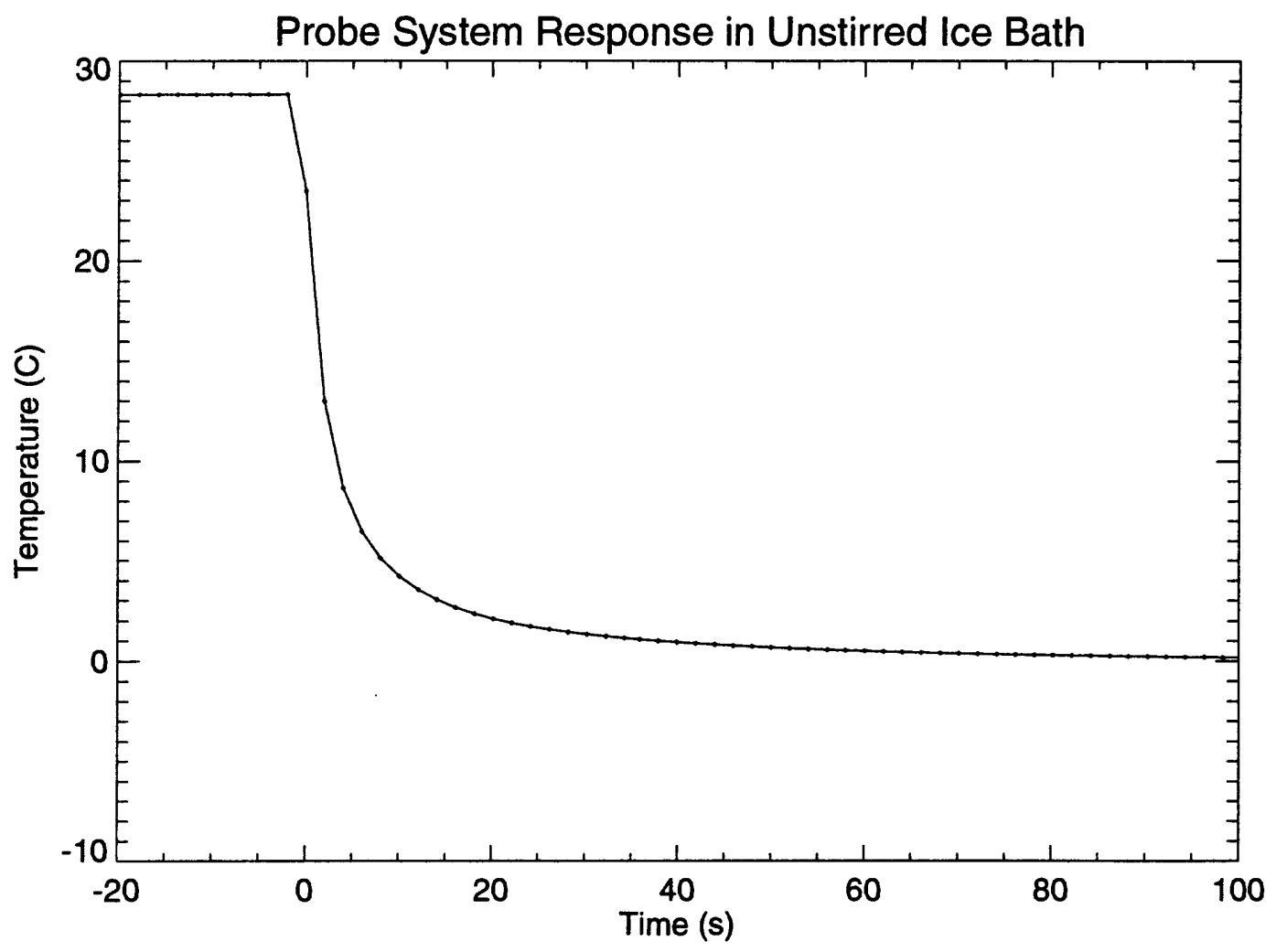

Time Constant in Unstirred Ice Bath

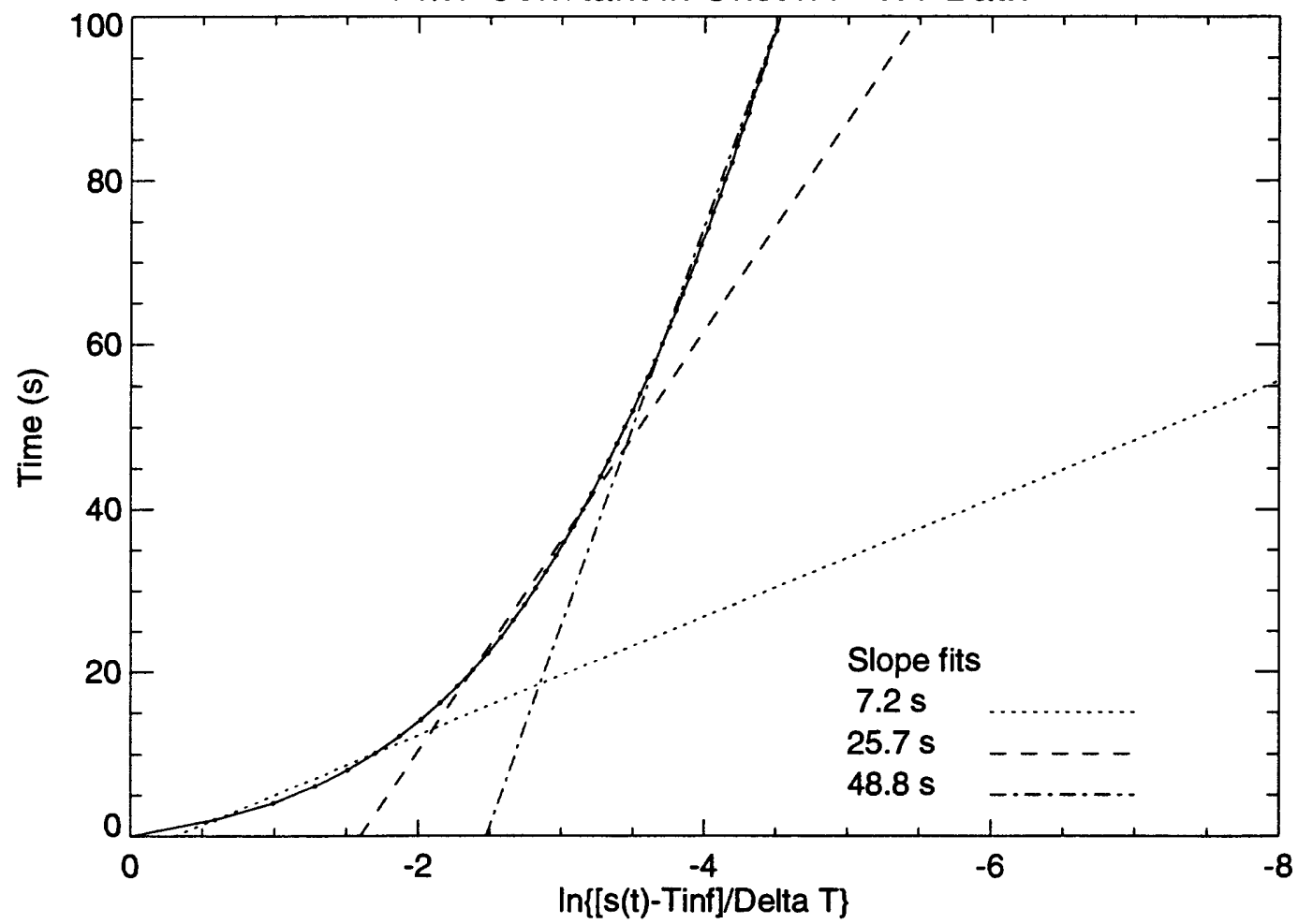

Figure A3: (top) Temperature verses time plot for Fenwal 212E thermister probe immersion in an ice bath at $0^{\circ} \mathrm{C}$. (bottom) Time verses $\ln \left[\left(s(t)-T_{\infty}\right) / \Delta T\right]$ plot of Fenwal $212 \mathrm{E}$ thermister probe response in an ice bath at $0^{\circ} \mathrm{C}$. Initial time constant $=7.2 \mathrm{~s}$. 


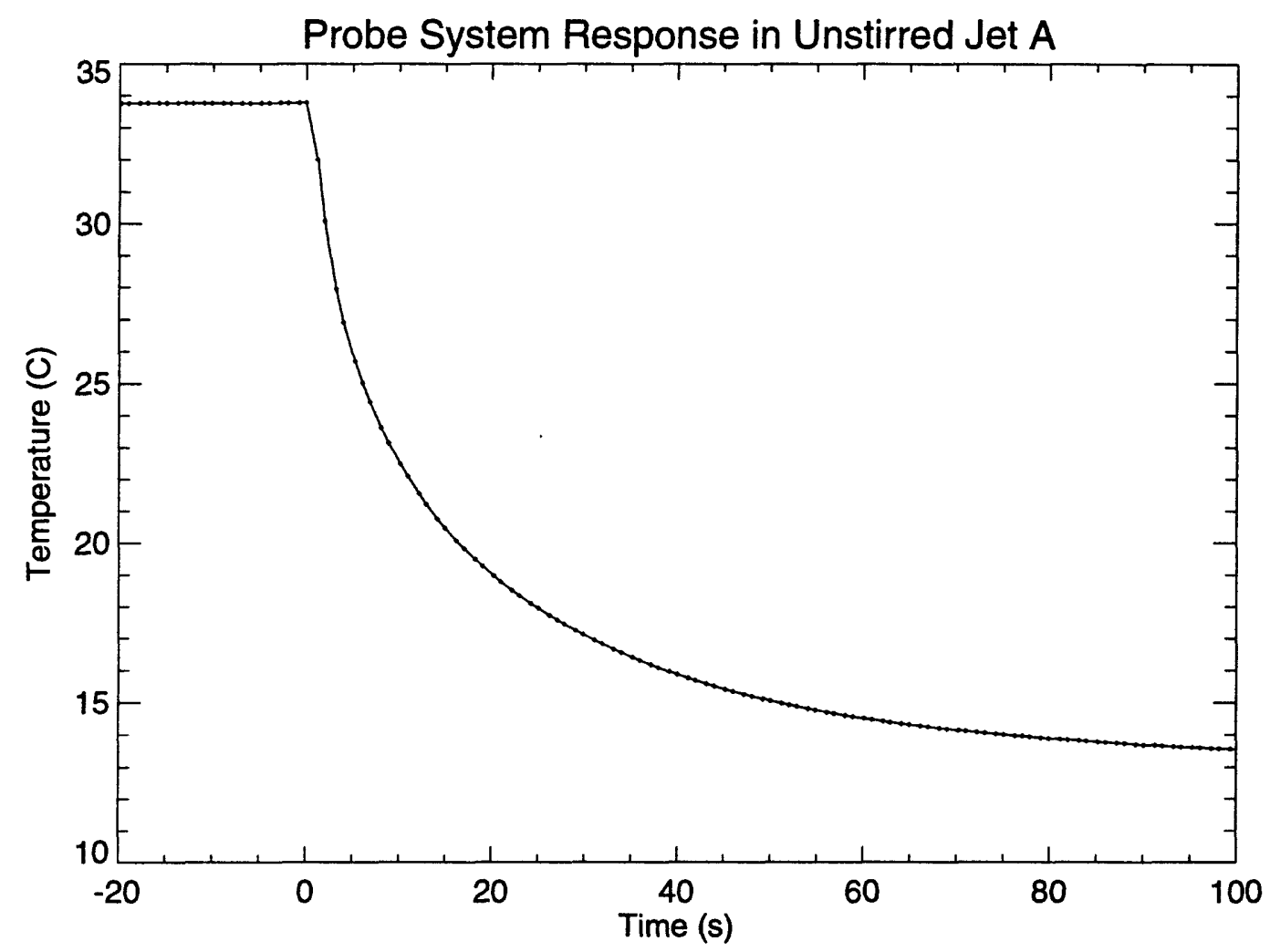

Time Constant in Unstirred Jet A

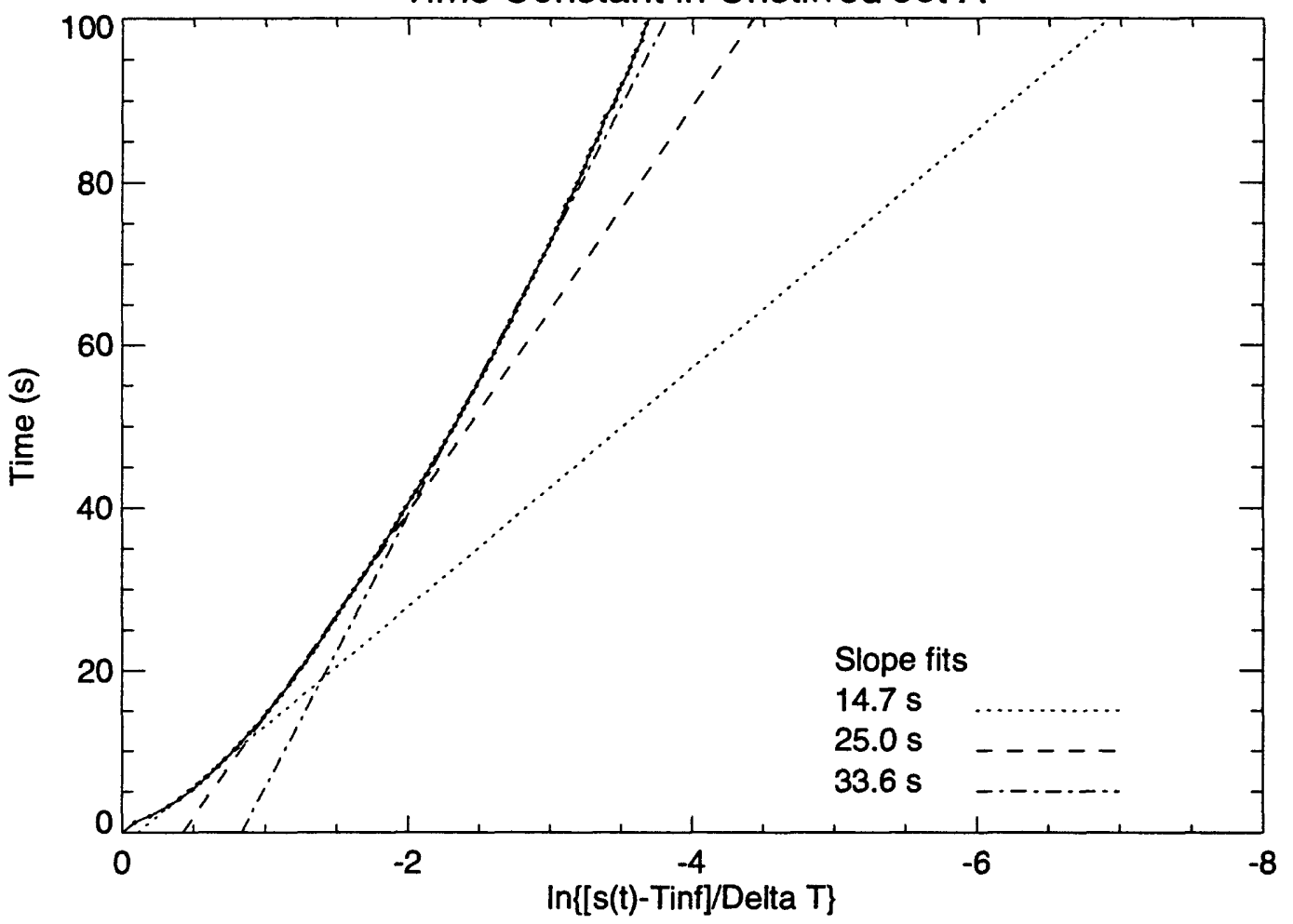

Figure A4: (top) Temperature verses time plot for Fenwal 212E thermister probe immersion in still Jet $\mathrm{A}$ fuel at $13{ }^{\circ} \mathrm{C}$. (bottom) Time verses $\ln \left[\left(s(t)-T_{\infty}\right) / \Delta T\right]$ plot of Fenwal $212 \mathrm{E}$ thermister probe response in still Jet $\mathrm{A}$ fuel at $13{ }^{\circ} \mathrm{C}$. Initial time constant $=14.7 \mathrm{~s}$. 


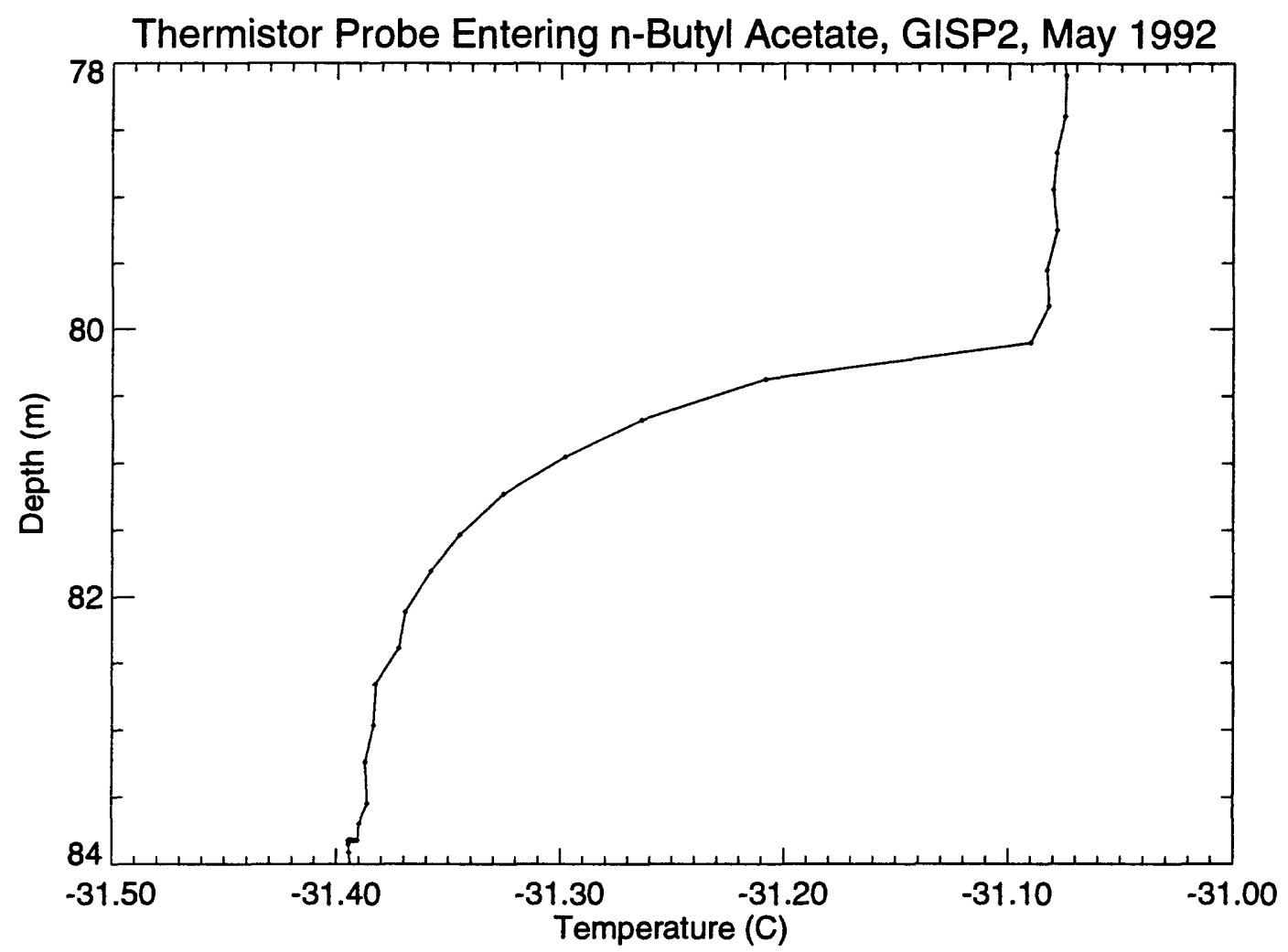

Figure A5: Depth verses temperature plot for Fenwal 212E thermister probe immersion in n-butyl acetate at GISP2, May 1992. 

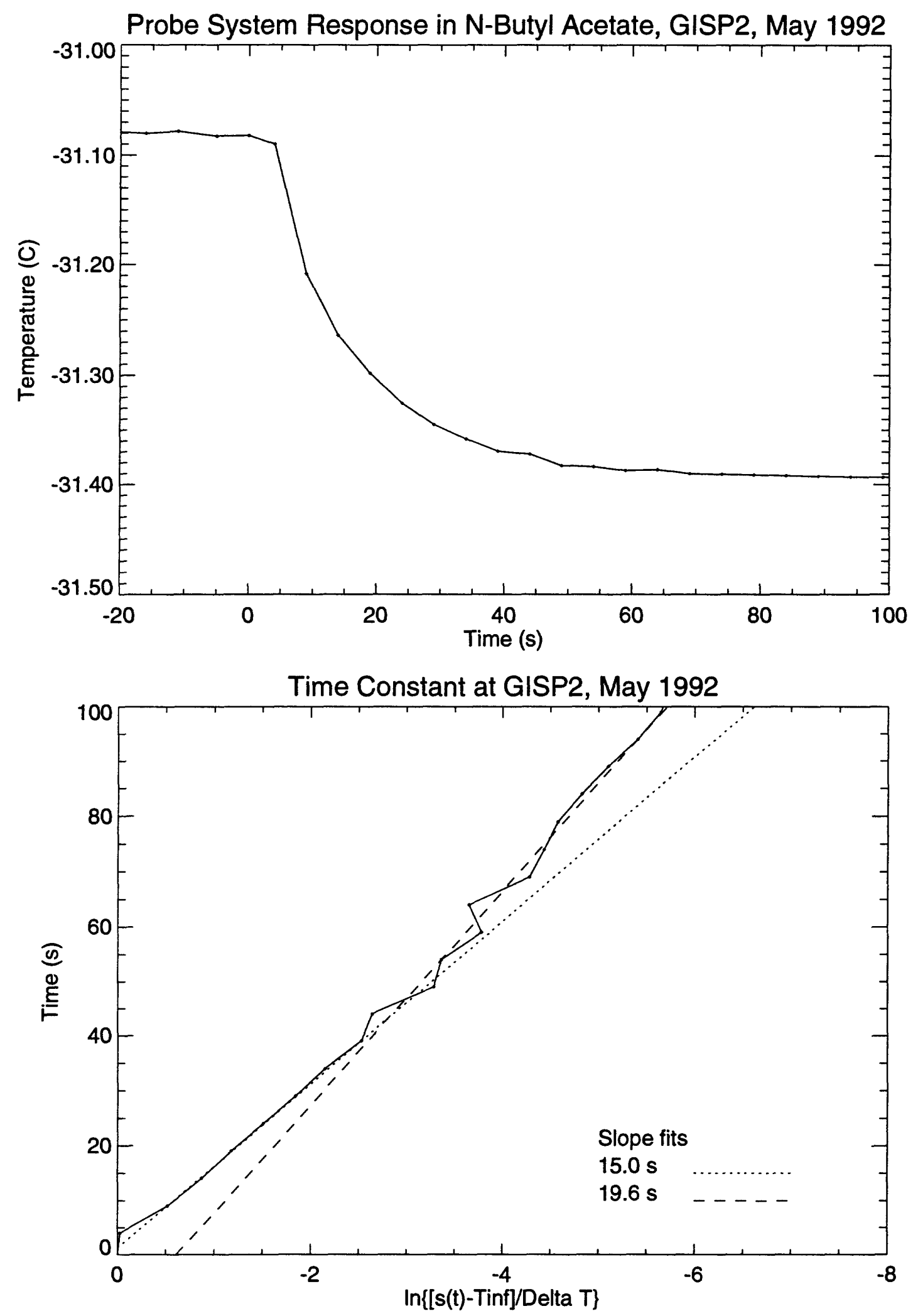

Figure A6: (top) temperature verses time plot for Fenwal 212E thermister probe immersion in n-butyl acetate at GISP2, May 1992. (bottom) Time verses $\ln \left[\left(s(t)-T_{\infty}\right) / \Delta T\right]$ plot of Fenwal 212E thermister probe immersion in n-butyl acetate at GISP2, May 1992. Initial time constant $=15 \mathrm{~s}$. 


\section{APPENDIX B: COMPUTER CODES}

The following computer codes, written in standard FORTRAN-77 and in IDL Version 3.1 (Research Systems, Inc., 1993), were used for the filtering and deconvolution described in this report.

8.1 convol.f - discrete probe response function

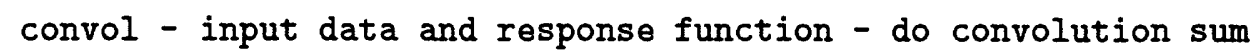




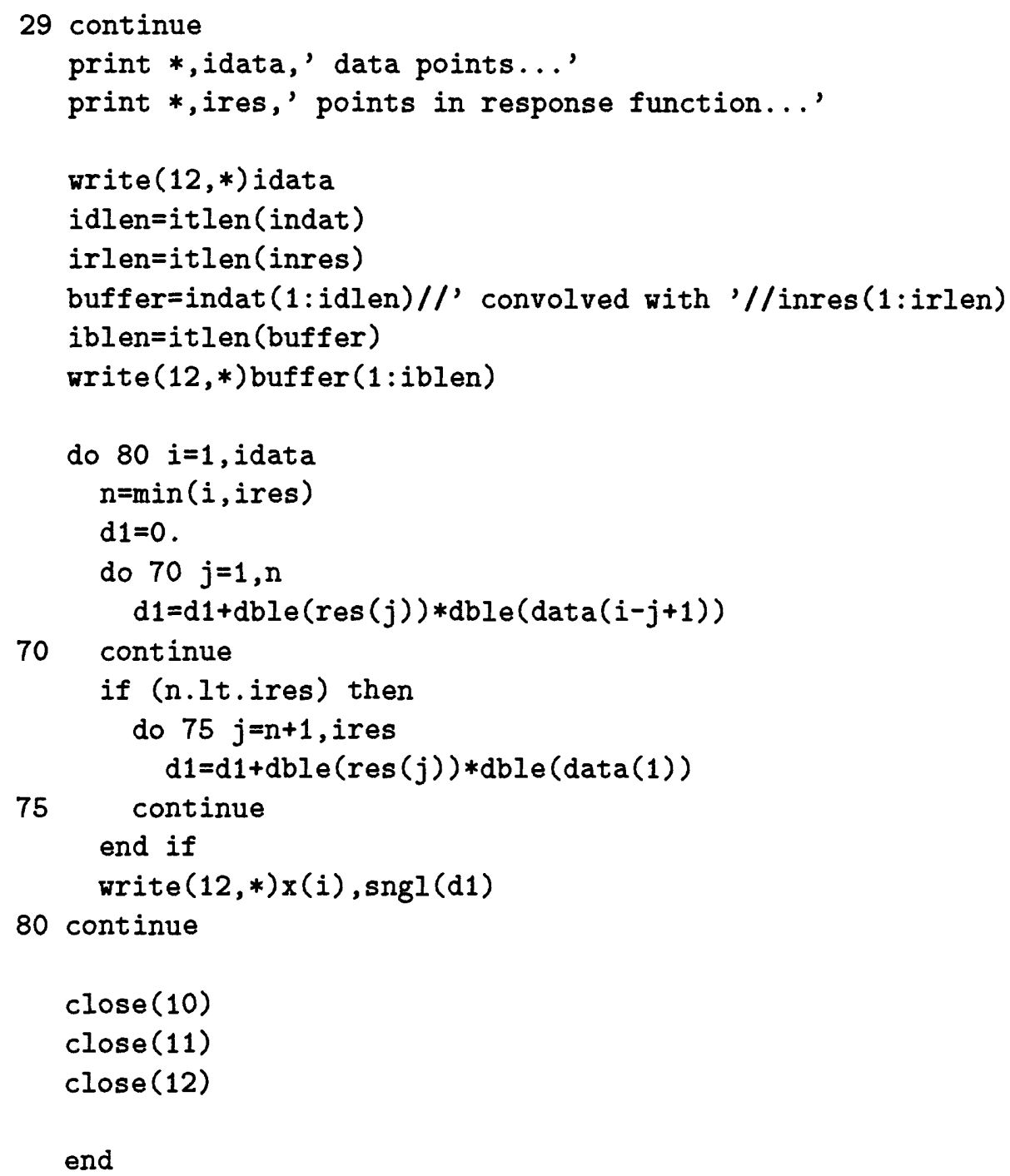

\section{2 response.f - discrete probe response function}

c

c response.f - make a discrete probe impulse response function for use

C with deconvolution tests

C

c

program response

dimension res (8192)

character $* 80$ outfile 


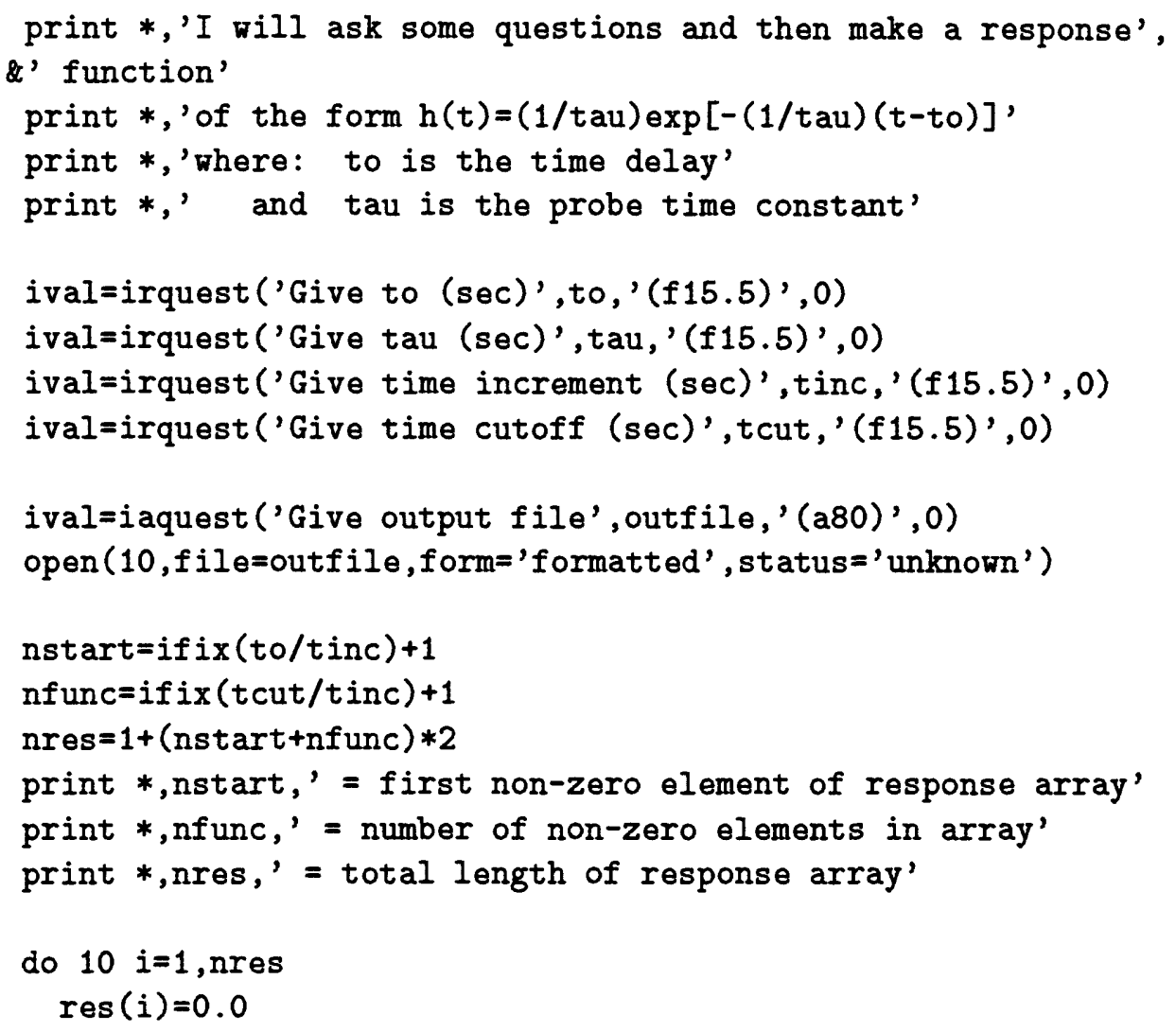

10 continue

sum $=0.0$

rat $=$ tinc/tau

do $20 i=0$, nfunc -1

c

$\operatorname{res}($ nstart $+i)=1 . / \operatorname{tau} * \exp ((-1 . /$ tau $) *($ float $(i) * \operatorname{tinc}))$

$\operatorname{res}($ nstart $+i)=\operatorname{rat} * \exp (-\operatorname{rat} *(f l o a t(i)))$

sum $=$ sum+res (nstart $+i$ )

20 continue

truesum $=1-\exp (-t c u t / t a u)$

print *, 'actual function sum $=$ ', sum

print $*$,' true sum value $=$ ', truesum

c normalize to 1

sumtest $=0$.

do $30 i=0$, nfunc-1

res (nstart $+i)=$ res (nstart $+i) /$ sum sumtest=sumtest+res (nstart $+i$ )

30 continue

print *,'normalized function sum $=$ ', sumtest 


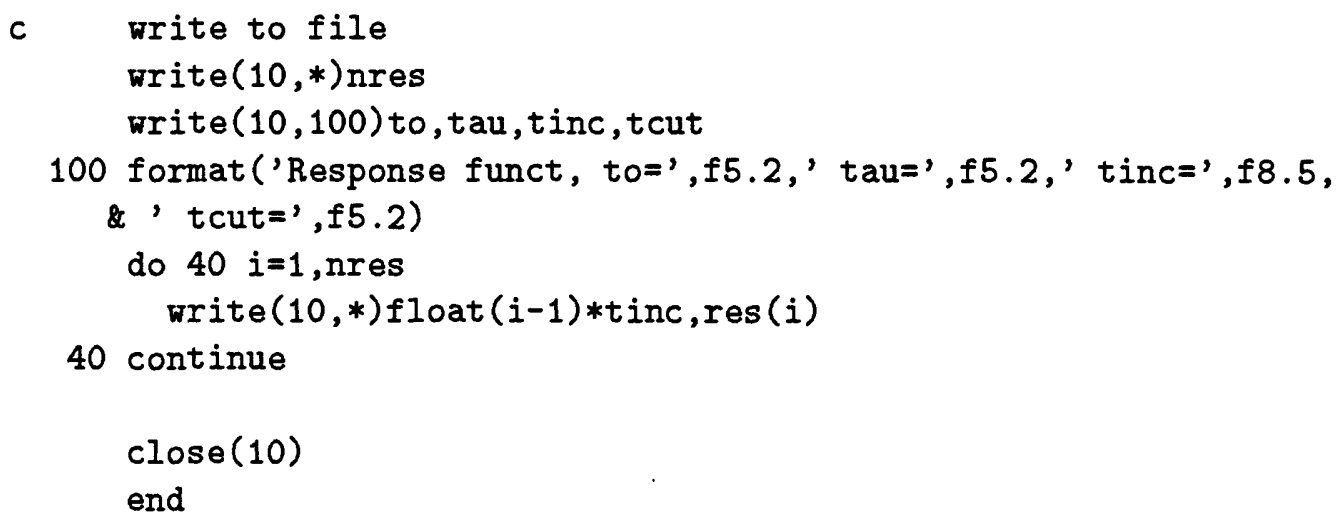

\subsection{Example IDL program for optimal filtering}

; Optimal smoothing of May 1992 GISP2 temperature log

Richard W. Saltus - for climate project

$a=$ temperature data

$b=$ flipped temperature array for FFT purposes

$a b=a$ concatenated with $b$

; $c=$ calculated power spectrum of $a b$

construct a continuous, repeating function for FFT analysis

$a=$ ydat

ashift $=a(0)$

aprime $=a-$ ashift

ntemp $=$ indgen(nd1)

aslope $=\operatorname{aprime}($ nd1-1)/ntemp (nd1-1)

atilt = aprime - aslope*ntemp

;

$b=$ reverse(atilt); flip a and put in $b$

bprime $=-1 * b$

abprime $=[$ atilt, $\operatorname{bprime}(1: n d 1-2)]$; concatenate $a$ and $b$ into $a b$

$n=n d 1 * 2$; create array $(f)$ of frequencies

$t=2$.

$\mathrm{n} 21=\mathrm{n} / 2+1$

$f=$ indgen $(n)$

$f(n 21)=n 21-n+f i n d g e n(n 21-2)$

$f=f /(n * t)$

$c=\left(\operatorname{abs}(F F T(\text { abprime, }-1))^{-2}\right)$; calculate power spectrum of $c$ $c \log =\operatorname{alog} 10(c)$

;

; calculate signal and noise functions 


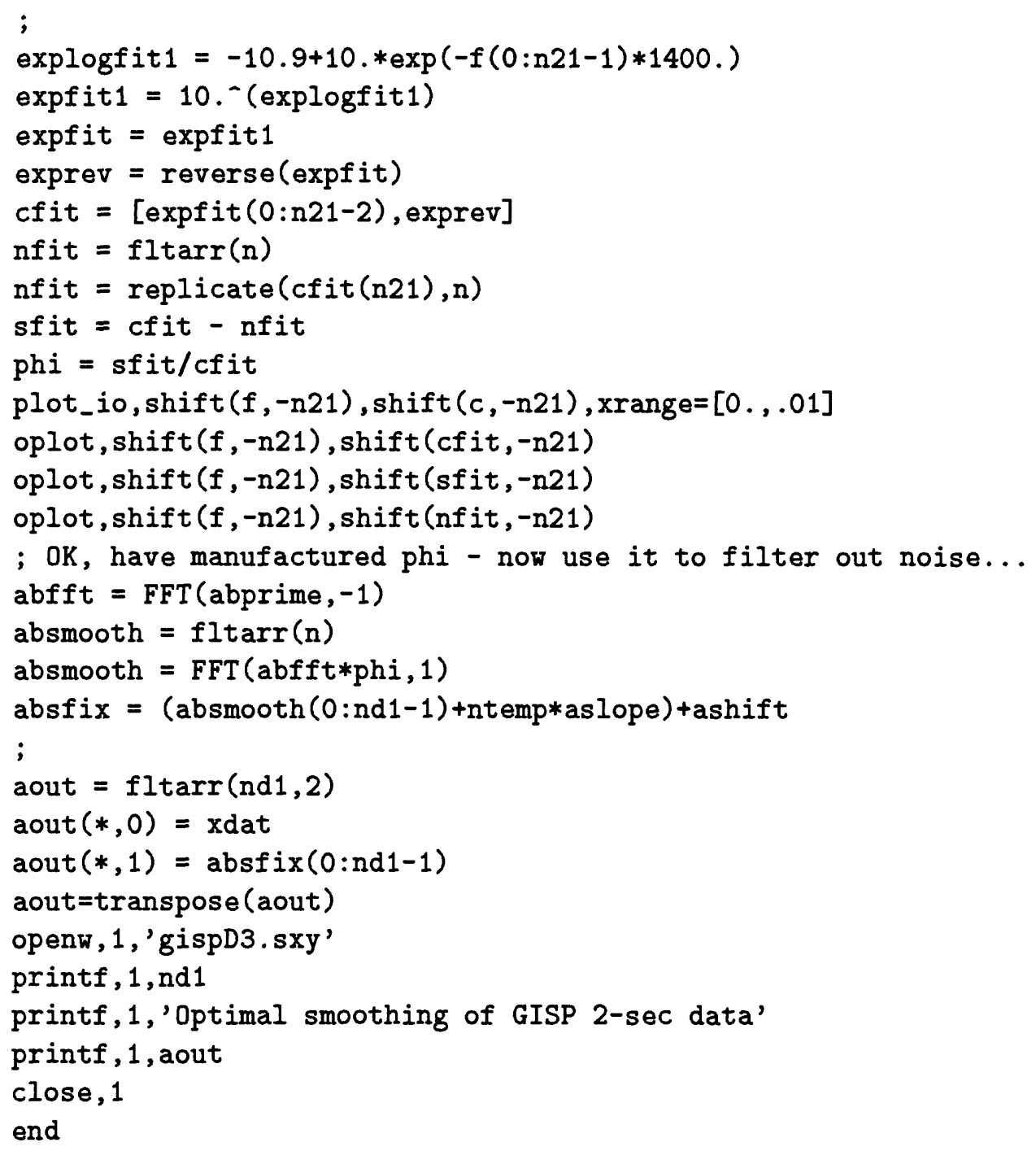

\section{4 simdecon.f - exact discrete deconvolution}

C

c simdecon - input convolution and response function - invert for decon

C

c R.W. Saltus - U.S. Geological Survey

c

C This program uses "serial division" (see Bracewell, 1986,

c The Fourier Transform and Its Applications, McGran_Hill,

c chapter 3) to deconvolve a measured function (data) and an

c assumed response function (res) to obtain a signal function (s).

c

c This form of deconvolution depends on the measurement apparatus

c starting at an equilibrium value (i.e., measurement(1) $=\operatorname{signal}(1)$. 
c

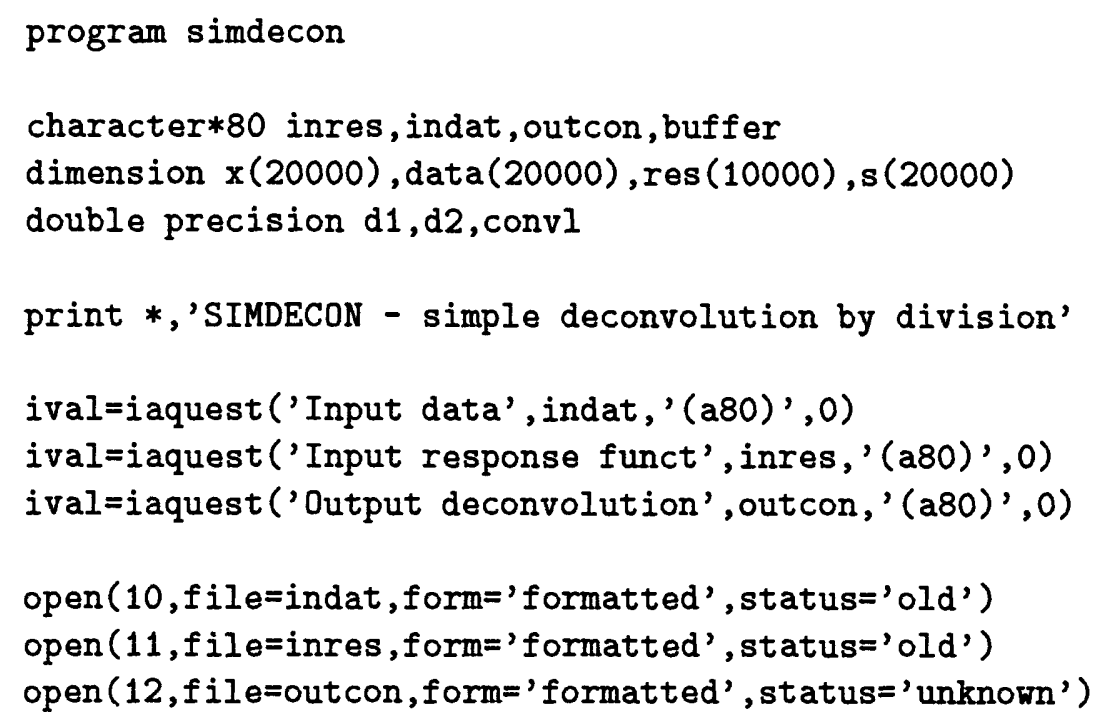

c read the measured function into the data array

c number of measurements = idata

$\operatorname{read}(10, *) \mathrm{n}$

$\operatorname{read}\left(10, '(a 80)^{\prime}\right)$ buffer

print *, buffer

idat $a=0$

10 continue

$\operatorname{read}(10, *$, end $=15) \times($ idata +1$)$, data $($ idata +1$)$

idata $=$ idat $a+1$

goto 10

c read the response function into res, number of points = ires

15 continue

$\operatorname{read}(11, *) \mathrm{n}$

$\operatorname{read}\left(11, '(a 80)^{\prime}\right)$ buffer

print *, buffer

ires $=0$

20 continue

$\operatorname{read}(11, *$, end $=30) z, \operatorname{res}($ ires +1$)$

ires $=$ ires +1

goto 20

30 continue 
close(11)

close(12)

end 